\title{
On the efficiency of plasma heating by Pedersen current dissipation from the photosphere to the lower corona
}

\begin{abstract}
M. L. Goodman`
Institute for Scientific Research, 2500 Fairmont Avenue - Suite 734, Fairmont, WV 26555-2720, USA

Received 11 June 2003 / Accepted 2 December 2003

Abstract. A model is presented that uses the electrical conductivity tensor of a multi-species plasma to estimate the efficiency $Q$ of plasma heating by Pedersen current dissipation as a function of height from the photosphere to the lower corona. The particle densities and temperature are given by FAL model CM. $Q$ is the efficiency with which the electric field generates thermal energy by transferring energy to the current density $\boldsymbol{J}_{\perp}$ perpendicular to the magnetic field. The energy is then thermalized by collisions. The projection of $\boldsymbol{J}_{\perp}$ on the driving electric field is the Pedersen current density. $Q$ is the ratio of the actual heating rate due to Pedersen current dissipation to the heating rate when $\boldsymbol{J}_{\perp}$ is entirely a Pedersen current, which is the maximum possible heating rate for given $\boldsymbol{J}_{\perp}$. It is found that Pedersen current dissipation is highly efficient throughout the chromosphere, but is highly inefficient in the transition region and corona on the spatial scales of FAL CM. In the photosphere, the electron magnetization, which is the product of the cyclotron frequency and the collision time is so small compared to unity that the conductivity tensor is almost isotropic, implying there is no essential difference between Pedersen current dissipation and magnetic field aligned current dissipation. It is the rapid increase with height of the magnetizations of electrons, protons and metallic ions from $\lesssim 1$ to $\gg 1$ beginning near the height of the FAL CM temperature minimum that causes Pedersen current dissipation to become essentially different from magnetic field aligned current dissipation, and that causes $Q$ to rapidly increase from minimum values $\sim 0.1$ near the temperature minimum to $\sim 1$ in the lower chromosphere. $Q$ remains $\sim 1$ up to the transition region in which it precipitously decreases with height to values $\lesssim 10^{-10}$ in the corona. It is proposed that the rapidly increasing magnetization triggers the onset of heating by Pedersen current dissipation that causes the chromospheric temperature inversion and heats the entire non-flaring chromosphere. The energy channeled by any mechanism into the generation of a center of mass (CM) electric field that drives current perpendicular to the magnetic field is thermalized by Pedersen current dissipation at the maximum possible rate throughout the chromosphere. The mechanism is damped in the chromosphere to the degree to which its energy is channeled into the creation of the CM electric field. The results of the model are consistent with previous predictions that slow magnetoacoustic waves heat network regions of the chromosphere through dissipation of Pedersen currents driven by a wave generated convection electric field, and that electric current dissipation on the spatial scales of the FAL models is insignificant for heating the transition region.
\end{abstract}

Key words. Sun: chromosphere - Sun: transition region - Sun: corona - MHD - Sun: magnetic fields - stars: atmospheres

\section{Introduction}

The net radiative loss from the solar chromosphere is $\sim 10^{7} \mathrm{ergs} \mathrm{cm}^{-2} \mathrm{~s}^{-1}$. This loss, and hence the chromospheric heating rate, is 10 times that of the quiet transition region and corona combined. It is an open question as to whether the corona is heated homogeneously, or inhomogeneously in hot loops. Observations by Aschwanden (2001) that support the latter view indicate that regions of the corona permeated by magnetic field lines that are connected to active regions contain plasma of chromospheric origin, that these regions emit $\sim 80 \%$ of the coronal net radiative loss near solar maximum, and that this radiative loss is localized at heights $\lesssim 10^{4} \mathrm{~km}$ above the photosphere. These observations suggest that the

* Also with the Department of Physics, The Catholic University of America, Washington, DC, USA.

e-mail: mgoodman@isr.us corona is heated primarily near its base. If this is true, then chromospheric processes might play an important role in coronal heating.

This paper presents magnetohydrodynamic (MHD) model evidence that Pedersen current dissipation is a highly efficient mechanism for dissipating energy in electric currents that flow orthogonal to the magnetic field in non-flaring regions of the chromosphere. The model is based on the electrical conductivity tensor of a multi-species plasma derived under the assumption that the single particle distribution functions are close to Maxwellian. This assumption is probably valid from the photosphere to the lower corona, defined as the region where the temperature $T \lesssim 10^{6} \mathrm{~K}$, and the density $n \gtrsim 10^{9} \mathrm{~cm}^{-3}$. For a detailed discussion of the conditions under which this assumption is valid see Sect. 2.1 of Goodman (1998) and the references cited therein. The validity of the assumption increases as 
the temperature decreases, and as the density increases. Under this assumption an accurate description of transport processes is given by the classical transport coefficients (Braginskii 1965; Chapman \& Cowling 1970; Mitchner \& Kruger 1973; Balescu 1988). An accurate description of the relevant transport processes must be included in MHD models in order to reliably model MHD heating and flow generation mechanisms. The classical transport coefficients vary by orders of magnitude with height from the photosphere to the corona, and are anisotropic in the presence of a magnetic field. They are tensors for electrical and thermal conduction, viscosity, and thermoelectric effects.

The net radiative losses from the network, internetwork, and non-flaring active regions of the chromosphere are all $\sim 10^{7} \mathrm{ergs} \mathrm{cm}^{-2} \mathrm{~s}^{-1}$. The primary difference between these regions is the photospheric filling factor of magnetic field concentrations, and their maximum field strengths. For these regions these quantities are largest in active regions, and smallest in internetwork. This property of the photospheric magnetic field is directly correlated with the chromospheric net radiative loss. Magnetic field concentrations in the photosphere remain unresolved on the smallest observable spatial scales of $\sim 0.5^{\prime \prime}$ for magnetograms (Domínguez Cerdeña et al. 2003), and $\sim 0.2^{\prime \prime}$ for bright points associated with kilogauss magnetic field concentrations (Lites et al. 1999). The magnetic field is concentrated in the boundary regions of the granulation, and the most intense heating in the overlying chromosphere is cospatial with the strongest magnetic field concentrations in these regions. These facts suggest that a single mechanism heats the network, internetwork, and non-flaring active regions of the chromosphere, and that it is an MHD mechanism.

An MHD mechanism for heating the chromospheric network is proposed by Goodman $(2000,2001)$. The mechanism involves dissipation of Pedersen currents driven by the convection electric field generated by slow magnetoacoustic waves. Here it is argued that Pedersen current dissipation is an efficient mechanism for dissipating the energy in electric current that is driven orthogonal to the magnetic field by any process that operates in non-flaring regions of the chromosphere. Such a process is damped to the degree to which its energy is channeled into driving this current. Examples of such processes are waves, quasi-steady flow across magnetic field lines, and possibly current sheet dynamics.

As observational resolution of the magnetic field in the photosphere has increased, the observed strength and number of magnetic field concentrations in the internetwork have also increased (Domínguez Cerdeña et al. 2003), and semiempirical modeling suggests that kilogauss strength magnetic fields localized in regions with sub-resolution spatial dimensions $\left(\lesssim 0.2^{\prime \prime}\right)$ exist in the internetwork, and account for most of the unsigned magnetic flux through the photosphere (see references in Sect. 1.1). This raises the possibility that magnetic field concentrations exist in the internetwork in sufficient strength and number to support an MHD mechanism for heating this region of the atmosphere. A summary of observational and theoretical evidence that the internetwork chromosphere is heated by an MHD mechanism is given in Sect. 1.1.
MHD mechanisms play a major role in heating the network and active regions of the chromosphere, transition region, and corona. This follows from the strong correlation between magnetic field strength and filling factor, and net radiative loss. An active region may be divided into flaring and non-flaring regions. Flare generation mechanisms are not considered here. However, anisotropic electrical conductivity, and the associated Pedersen current dissipation might play an important role in flare dynamics.

\subsection{Magnetic fields and heating in the internetwork chromosphere}

Ca II bright grains are one signature of heating in the internetwork. Observations by Carlsson et al. (1997) and Judge \& Carpenter (1998) suggest that although the formation of the $\mathrm{Ca}$ II $\mathrm{H}$ and $\mathrm{K}$ bright grains in the internetwork is probably due to a hydrodynamic shock wave heating mechanism similar to the one proposed by Carlsson \& Stein (1997), the amount of heating associated with these grains is more than an order of magnitude less than what is required to heat the internetwork chromosphere. A correlation between regions of high magnetic flux density ( $\gtrsim 4 \mathrm{Mx} \mathrm{cm}^{-2}$ ) and Ca II bright grains in the lower internetwork chromosphere is indicated by the observations of Sivaraman \& Livingston (1982) and Sivaraman et al. (2000). Observations of Lites et al. (1999) and Worden et al. (1999) suggest that magnetic fields are not necessary for the production of the internetwork Ca II bright grains, consistent with the accurate reproduction of spectral properties of these grains by the simulations of Carlsson \& Stein (1997). About 5-10\% of these grains occur with an intensity enhanced by a factor $\sim 3-4$ at sites of enhanced magnetic field strength, which are probably sites of strong $(\sim 500 \mathrm{G})$ photospheric flux tubes of the type observed by Lin (1995) (Nindos \& Zirin 1998; Lites et al. 1999; Krijger et al. 2001), suggesting that the presence of the magnetic field amplifies an otherwise hydrodynamic heating mechanism. Sivaraman et al. (2000) emphasize the importance of including bipolar magnetic regions, in addition to apparently unipolar regions when testing for a correlation between bright grains and magnetic fields. By including these bipolar regions, Sivaraman et al. (2000) establish an $85 \%$ spatial correlation between bright grains and magnetic fields. The grains are not isolated heating phenomena, but occur as localized enhancements over background $\mathrm{Ca}$ II intensity and velocity oscillations that are coherent over regions with characteristic horizontal dimensions $\sim 10^{3}-10^{4} \mathrm{~km}$, with the intensity of the brightest grains approaching that of network bright grains (von Uexküll \& Kneer 1995; Lites et al. 1999). The presence of magnetic fields amplifies the intensity of CaII grains, but the complete role of magnetic fields in grain formation is not clear.

The main mode of oscillation of the internetwork photosphere is the $\mathrm{p}$ mode oscillation. $\mathrm{p}$ modes have peak amplitude at periods $\sim 5 \mathrm{~min}$. They drive chromospheric oscillations that have peak amplitudes at periods $\sim 2-8 \mathrm{~min}$, horizontal coherence lengths $\sim 2000-8000 \mathrm{~km}$, a vertical structure and dynamics that is accurately reproduced by the simulations of Carlsson \& Stein (1997), and that are strongly modified by the presence 
of internetwork magnetic fields (Hoekzema et al. 1997; Curdt \& Heinzel 1998; Lites et al. 1999; Wikstol et al. 2000; Judge et al. 2001; Krijger et al. 2001).

Outside of CaII bright grains, a role for the magnetic field in internetwork heating is suggested by observations of internetwork magnetic fields in the photosphere that indicate the presence of 200-1300 G magnetic flux tubes over $\sim 15 \%$ of the observation area (Grossman-Doerth et al. 1996), sparsely distributed $500 \mathrm{G}$ magnetic flux tubes (Lin 1995; Keller et al. 1994), and a weaker field component 200-500 G distributed uniformly over the boundaries of the granulation (Lin \& Rimmele 1999; Solanki et al. 1996). This implies the presence of magnetic fields in the overlying chromosphere that may play a role in heating.

Observations and semi-empirical modeling suggest that most of the magnetic flux through the photosphere in network, internetwork, plage, and sunspot penumbrae is not detected, that magnetic fields with strengths $\sim 10^{2}-10^{3} \mathrm{G}$ exist on sub-resolution scales with flux tube diameters as small as a few kilometers, and that these fields are often inferred to be weak fields (Sánchez Almeida \& Landi degl'Innocenti 1996; Sánchez Almeida et al. 1996; Sánchez Almeida 1997, 2000; Sánchez Almeida \& Lites 2000; Muller et al. 2000; Sánchez Almeida et al. 2001; Berger \& Title 2001; Socas-Navarro \& Sánchez Almeida 2002; Domínguez Cerdeña et al. 2003). This inference is based on an assumed plasma state that is a combination of the relatively low plasma density in, and hence low emission from, regions of relatively high magnetic field strength, the embedding of these strong field regions in relatively weak field, denser and strongly emitting plasma in a resolution element, and the presence of mixed magnetic field polarities and geometries in a resolution element. If such widespread, sub-resolution strong magnetic fields exist, they are potential sites for the excitation of MHD waves over most of the surface of the Sun. Granular dynamics is one mechanism for exciting such waves by interacting with magnetic flux tubes in granular boundaries (Kalkofen 1997; Hasan \& Kalkofen 1999; Hasan et al. 2000). Another possible wave excitation mechanism is a photospheric dynamo. MHD simulations suggest that convection on mesogranular, granular, and sub-resolution scales drives a turbulent dynamo that generates a randomly oriented magnetic field with magnitudes $\sim 10-10^{2} \mathrm{G}$ (Durney et al. 1993; Petrovay \& Szakaly 1993; Cattaneo 1999; Emonet \& Cattaneo 2001; Cattaneo et al. 2001). There is observational evidence for the existence of these turbulent fields (Stenflo 1982; Faurobert-Scholl 1993, 1994; Faurobert-Scholl et al. 1995; Stenflo et al. 1998; Bianda et al. 1998; Faurobert et al. 2001; Stenflo et al. 2001; Stenflo et al. 2002; Lites 2002), and for the existence of MHD waves in the network and internetwork chromosphere (Curdt \& Heinzel 1998; Krijger et al. 2001; McIntosh et al. 2001; McAteer et al. 2002), as well as in photospheric network and plage (Sigwarth 2001). These results suggest that many strong magnetic field regions in the internetwork are not detected, that turbulent granular convection excites MHD waves in these regions, and that photospheric dynamo action generates weaker turbulent magnetic fields that also excite MHD waves in these strong field regions, believed to be generated by the Sun's internal dynamo localized in a velocity shear layer between the radiative and convection zones. These observations and results from semi-empirical models suggest that the dissipation of these waves is an important source of chromospheric heating over the entire surface of the Sun outside of flaring regions.

The existence of magnetic field concentrations with magnitudes $\sim 10^{2}-10^{3} \mathrm{G}$ over most of the solar surface also suggests that the localized current distributions that must exist in the presence of these localized fields make an important contribution to atmospheric heating through Pedersen current dissipation. For example, azimuthal currents must exist in the presence of a vertical magnetic flux tube. These currents flow orthogonal to the magnetic field, and so are subject to some degree of Pedersen current dissipation.

\subsection{Elements of resistive heating}

Any resistive heating mechanism is based on the electric field transferring energy to the current density that is dissipated at a rate determined by collisional or collisionless processes. In the MHD model of a plasma these processes are represented by a conductivity tensor $\bar{\sigma}$.

The resistive heating rate per unit volume is

$q=\boldsymbol{J} \cdot \boldsymbol{E}^{\prime}$

where

$\boldsymbol{E}^{\prime}=\boldsymbol{E}+\frac{\boldsymbol{U} \times \boldsymbol{B}}{c}$

Here $\boldsymbol{J}, \boldsymbol{E}, \boldsymbol{B}, \boldsymbol{U}, \boldsymbol{E}^{\prime}$, and $c$ are the current density, electric and magnetic fields, center of mass (CM) velocity of the plasma, the electric field in the CM frame of reference, and the speed of light. $q$ is the portion of the work done by the electric field per unit volume and time on the charged particles that goes into increasing the thermal energy of the plasma. The remainder of the work done by the electric field goes into changing the $\mathrm{CM}$ kinetic energy of the plasma through the magnetic force in the momentum equation for the CM motion. The total work done by the electric field is, of course, $\boldsymbol{J} \cdot \boldsymbol{E}$.

The simplest form of Ohm's law that describes anisotropic resistive heating, and the one used here, is

$$
\begin{aligned}
\boldsymbol{J} & =\bar{\sigma} \cdot \boldsymbol{E}^{\prime} \\
& =\sigma_{\|} \boldsymbol{E}_{\|}+\sigma_{\mathrm{P}} \boldsymbol{E}_{\perp}^{\prime}+\sigma_{\mathrm{H}} \boldsymbol{E}^{\prime} \times \hat{\boldsymbol{B}}
\end{aligned}
$$

where $\boldsymbol{E}_{\perp}^{\prime}$ is the electric field perpendicular to $\boldsymbol{B}$ in the CM frame of reference, $\boldsymbol{E}_{\|}$is the electric field parallel to $\boldsymbol{B}, \hat{\boldsymbol{B}}$ is a unit vector in the direction of $\boldsymbol{B}$, and $\sigma_{\|}, \sigma_{\mathrm{P}}$, and $\sigma_{\mathrm{H}}$ are the total parallel, Pedersen, and Hall conductivities. The corresponding components of $\boldsymbol{J}$ are the parallel, Pedersen, and Hall current densities.

From the Ohm's law (4), and Eq. (1) it follows that

$$
\begin{aligned}
q & =\sigma_{\|} E_{\|}^{2}+\sigma_{P}{E_{\perp}^{\prime}}^{2} \\
& \equiv q_{\|}+q_{\mathrm{P}} .
\end{aligned}
$$

$q_{\|}$and $q_{\mathrm{P}}$ are the heating rates due to dissipation of magnetic field aligned and Pedersen currents. The Hall current is not dissipative since it is orthogonal to $\boldsymbol{E}^{\prime}$. 
In this paper, heating due to dissipation of magnetic field aligned currents is neglected. Partial justification for doing this is the prediction in Goodman (2000) that in the lower chromospheric network the heating rate due to dissipation of these currents when they are driven by linear MHD waves is $\sim 10^{6.5}-10^{9.5}$ times smaller than that due to Pedersen current dissipation. The relationship between $q_{\mathrm{P}} / q_{\|}$and the degree to which a magnetic field is force-free is discussed in Sect. 6.

The Pedersen and Hall current densities are

$\boldsymbol{J}_{\mathrm{P}}=\sigma_{\mathrm{P}} \boldsymbol{E}_{\perp}^{\prime}$

$\boldsymbol{J}_{\mathrm{H}}=\sigma_{\mathrm{H}} \boldsymbol{E}_{\perp}^{\prime} \times \hat{\boldsymbol{B}}$.

Then, if $\boldsymbol{J}_{\perp}$ is the current density orthogonal to $\boldsymbol{B}$,

$J_{\perp}^{2}=J_{\mathrm{P}}^{2}+J_{\mathrm{H}}^{2}$,

and $q_{\mathrm{P}}$ may be written as

$$
\begin{aligned}
q_{\mathrm{P}} & =\frac{\sigma_{\mathrm{P}} J_{\perp}^{2}}{\sigma_{\mathrm{P}}^{2}+\sigma_{\mathrm{H}}^{2}} \\
& \equiv \frac{\sigma_{\mathrm{P}}^{2}}{\sigma_{\mathrm{P}}^{2}+\sigma_{\mathrm{H}}^{2}} q_{\mathrm{P}, \max }
\end{aligned}
$$

where $q_{\mathrm{P}, \max }$ is the maximum possible value of $q_{\mathrm{P}}$ for given $\boldsymbol{J}_{\perp}$, achieved when $\boldsymbol{J}_{\mathrm{P}}=\boldsymbol{J}_{\perp}$.

The efficiency $Q$ of Pedersen current dissipation is then

$$
\begin{aligned}
Q & =\frac{q_{\mathrm{P}}}{q_{\mathrm{P}, \max }} \\
& =\frac{1}{1+\left(\frac{\sigma_{\mathrm{H}}}{\sigma_{\mathrm{P}}}\right)^{2}} .
\end{aligned}
$$

$Q$ is the efficiency with which $\boldsymbol{E}_{\perp}^{\prime}$ transfers energy to $\boldsymbol{J}_{\perp}$ that is then thermalized by Coulomb collisions or wave particle scattering. In order to compute $q_{\mathrm{P}}$ it is necessary to use a more general model that computes $\boldsymbol{E}_{\perp}^{\prime}$. Although $Q$ does not give any indication of the value of $q_{\mathrm{P}}$, except when $Q=0$, its value indicates how efficiently the mechanism that generates $\boldsymbol{E}_{\perp}^{\prime}$ heats the atmosphere.

\subsection{Relation to previous work}

The model extends aspects of the model used in Goodman (2000) in the following way. The assumption of weak ionization, and the corresponding restriction that the electrical conductivity is due only to electron- $\mathrm{H}$, proton- $\mathrm{H}$, and ion- $\mathrm{H}$ collisions are removed. The model allows for a variable, arbitrary degree of ionization as a function of height, and includes charged-charged and charged-HeI particle collisions in the calculation of the conductivity tensor. Above the lower chromosphere, electron-proton collisions are important in determining the conductivity tensor, and completely dominate chargedneutral particle collisions from the upper chromosphere into the lower corona. This extension allows the calculation of $Q$ to be extended above the lower chromosphere into the corona.

\section{Model for the conductivity tensor}

A model is needed for the Hall and Pedersen conductivities in order to evaluate $Q$ for a multi-species plasma that characterizes the atmosphere from the photosphere to the lower corona. This model must be valid for a $\mathrm{H}-\mathrm{He}$ gas that ranges from weakly to fully ionized, and that contains trace concentrations of metallic ions. Such a model is derived in this section. The usefulness of the derived conductivity tensor extends beyond the calculation of $Q$ in this paper since the conductivity tensor does not depend on any assumptions about spatial or time dependence. It may be used in a model that depends on any number of dimensions and that is time dependent. The conductivity tensor depends on temperature, particle densities, and magnetic field strength. It may be used in any model that computes these quantities self consistently or takes them as inputs. It may play an important role in future MHD models that describe solar atmospheric dynamics.

\subsection{Conductivity tensor for a three species plasma}

Mitchner \& Kruger (1973) use an MHD model to derive the Ohm's law for a three component plasma consisting of electrons, neutrals, and singly charged ions. The degree of ionization is arbitrary. Neglecting electron pressure gradient effects, consistent with the assumed Ohm's law Eq. (3), the derived Ohm's law gives

$$
\begin{aligned}
\sigma_{\|} & =\sigma_{\|}^{(\mathrm{e})} \\
\sigma_{\mathrm{P}} & =\frac{\sigma_{\|}(1+\Gamma)}{(1+\Gamma)^{2}+M_{\mathrm{e}}^{2}} \\
\sigma_{\mathrm{H}} & =\frac{-M_{\mathrm{e}} \sigma_{\|}}{(1+\Gamma)^{2}+M_{\mathrm{e}}^{2}} \\
\sigma_{\|}^{(\mathrm{e})} & =\frac{n_{\mathrm{e}} \mathrm{e}^{2}}{m_{\mathrm{e}} v_{\mathrm{eh}}} \\
\Gamma & =\left(\frac{\rho_{\mathrm{n}}}{\rho}\right)^{2} M_{\mathrm{e}} M_{\mathrm{i}} \\
M_{\mathrm{e}} & =\frac{\omega_{\mathrm{e}}}{v_{\mathrm{eh}}} \\
M_{\mathrm{i}} & =\frac{\omega_{\mathrm{i}}}{v_{\mathrm{i}}^{*}} \\
\omega_{\mathrm{e}} & =\frac{e B}{m_{\mathrm{e}} c} \\
\omega_{\mathrm{i}} & =\frac{e B}{m_{\mathrm{i}} c} \\
v_{\mathrm{i}}^{*} & =\frac{m_{\mathrm{n}}}{m_{\mathrm{i}}+m_{\mathrm{n}}} v_{\mathrm{in}} \\
m_{\mathrm{in}} & =\frac{m_{\mathrm{i}} m_{\mathrm{n}}}{m_{\mathrm{i}}+m_{\mathrm{n}}} .
\end{aligned}
$$

Here $\sigma_{\|}^{(\mathrm{e})}, n_{\mathrm{e}}, e, m_{\mathrm{e}}, B, \rho, \rho_{\mathrm{n}}, v_{\mathrm{eh}}, v_{\mathrm{in}}, \omega_{\mathrm{e}}, m_{\mathrm{n}}, m_{\mathrm{i}}, m_{\mathrm{in}}, \omega_{\mathrm{i}}$, and $v_{\mathrm{i}}^{*}$ are the electron conductivity parallel to $\boldsymbol{B}$, electron number density, magnitude of the electron charge, electron mass, $|\boldsymbol{B}|$, total mass density, neutral particle mass density, electron-heavy particle collision frequency $=v_{\mathrm{en}}+v_{\mathrm{ei}}=$ electron-neutral collision frequency + electron-ion collision frequency, ion-neutral collision frequency, electron cyclotron frequency, neutral 
particle mass, ion mass, ion-neutral particle reduced mass, ion cyclotron frequency, and reduced ion collision frequency.

$M_{\mathrm{e}}$ and $M_{\mathrm{i}}$ are the magnetizations of electrons and ions. In general, for a plasma with any number of species the magnetization of any species $s$ is defined as

$$
\begin{aligned}
M_{s} & =\frac{\omega_{s}}{v_{s}^{*}} \\
\omega_{s} & =\frac{\left|q_{s}\right| B}{m_{s} c} \\
v_{s}^{*} & =\sum_{j \neq s} \frac{m_{j}}{m_{s}+m_{j}} v_{s j} \\
& \equiv \sum_{j \neq s} v_{s j}^{*}
\end{aligned}
$$

where $q_{s}, v_{s}^{*}, v_{s j}^{*}$, and $v_{s j}$ are the charge and total reduced collision frequency of species $s$, the reduced collision frequency of a particle of species $s$ with particles of species $j$, and the collision frequency of a particle of species $s$ with particles of species $j$. Since the electron mass is so much smaller than all other particle masses, $v_{\mathrm{e}}^{*} \sim \sum_{j} v_{\mathrm{e} j} \equiv v_{\mathrm{e}}$ which is the total collision frequency of electrons with all other species. The meaning of $v_{s}^{*}$ and $v_{s j}^{*}$ in terms of the rate of collisional momentum transfer to species $s$ is as follows. In the momentum equation for species $s$, the rate per unit volume at which collisions with species $j$ transfer momentum to species $s$ is

$\dot{\boldsymbol{P}}_{s j}=\rho_{s} v_{s j}^{*}\left(\boldsymbol{V}_{j}-\boldsymbol{V}_{s}\right)$,

where $\boldsymbol{V}_{j}$ and $\boldsymbol{V}_{s}$ are the fluid velocities of species $j$ and $s$, and $\rho_{s}$ is the mass density of species $s$. Then the rate per unit volume at which momentum is transferred to species $s$ by collisions with all unlike species is

$$
\begin{aligned}
\sum_{j \neq s} \dot{\boldsymbol{P}}_{s j} & =\rho_{s} v_{s}^{*}\left(\langle\boldsymbol{V}\rangle-\boldsymbol{V}_{s}\right) \\
& \equiv \sum_{j \neq s} \dot{\boldsymbol{p}}_{s j}
\end{aligned}
$$

where

$$
\langle\boldsymbol{V}\rangle=\frac{\sum_{j \neq s} v_{s j}^{*} \boldsymbol{V}_{j}}{\sum_{j \neq s} v_{s j}^{*}}
$$

is the reduced collision frequency weighted average fluid velocity of the unlike particle species, and

$\dot{\boldsymbol{p}}_{s j}=\rho_{s} v_{s j}^{*}\left(\langle\boldsymbol{V}\rangle-\boldsymbol{V}_{s}\right)$

is an average rate per unit volume at which collisions with species $j$ transfer momentum to species $s$. Equations (31)-(33) show that the total rate of momentum transfer to species $s$ may be written as a sum over momentum transfer rates from unlike species each of which has velocity $\langle\boldsymbol{V}\rangle$. Then $v_{s j}^{*}$ is the effective collision frequency for these momentum transfer rates, and $v_{s}^{*}$ is the corresponding effective total collision frequency. $v_{s j}^{*}$ and $v_{s}^{*}$ are called reduced collision frequencies since $v_{s j}^{*}<v_{s j}$.

Using Eqs. (15) and (16), Eq. (13) for the efficiency of heating due to Pedersen current dissipation becomes

$Q=\frac{1}{1+\left(\frac{M_{\mathrm{e}}}{1+\Gamma}\right)^{2}}$.
Given the total conductivity tensor defined by Eqs. (14)-(24), the electron and ion conductivity tensors are determined as follows.

Let $\boldsymbol{U}_{\mathrm{e}}, \boldsymbol{U}_{\mathrm{i}}$, and $\boldsymbol{U}_{\mathrm{n}}$ be the diffusion velocities of electrons, ions, and neutrals measured with respect to the center of mass velocity $\boldsymbol{U}$. Let $\boldsymbol{u}_{\mathrm{e}}, \boldsymbol{u}_{\mathrm{i}}$, and $\boldsymbol{u}_{\mathrm{n}}$ be the velocities of the electron, ion, and neutral fluids. Then, by definition,

$$
\begin{aligned}
\boldsymbol{U} & =\frac{\rho_{\mathrm{e}} \boldsymbol{U}_{\mathrm{e}}+\rho_{\mathrm{i}} \boldsymbol{U}_{\mathrm{i}}+\rho_{\mathrm{n}} \boldsymbol{U}_{\mathrm{n}}}{\rho} \\
\boldsymbol{U}_{\mathrm{e}} & =\boldsymbol{u}_{\mathrm{e}}-\boldsymbol{U} \\
\boldsymbol{U}_{\mathrm{i}} & =\boldsymbol{u}_{\mathrm{i}}-\boldsymbol{U} \\
\boldsymbol{U}_{\mathrm{n}} & =\boldsymbol{u}_{\mathrm{n}}-\boldsymbol{U}
\end{aligned}
$$

so that

$\rho_{\mathrm{e}} \boldsymbol{U}_{\mathrm{e}}+\rho_{\mathrm{i}} \boldsymbol{U}_{\mathrm{i}}+\rho_{\mathrm{n}} \boldsymbol{U}_{\mathrm{n}}=0$.

The total current density is the sum of the electron and ion current densities.

$$
\begin{aligned}
\boldsymbol{J} & =\boldsymbol{J}_{\mathrm{i}}+\boldsymbol{J}_{\mathrm{e}} \\
& =e\left(n_{\mathrm{i}} \boldsymbol{U}_{\mathrm{i}}-n_{\mathrm{e}} \boldsymbol{U}_{\mathrm{e}}\right)
\end{aligned}
$$

where $n_{s}$ is the number density of species $s$.

Solving Eqs. (39) and (41) for the electron and ion diffusion velocities gives

$$
\begin{aligned}
& \boldsymbol{U}^{(\mathrm{e})}=\frac{-m_{\mathrm{i}} \boldsymbol{J}-e \rho_{\mathrm{n}} \boldsymbol{U}_{\mathrm{n}}}{e n_{\mathrm{e}}\left(m_{\mathrm{e}}+m_{\mathrm{i}}\right)} \\
& \boldsymbol{U}^{(\mathrm{i})}=\frac{m_{\mathrm{e}} \boldsymbol{J}-e \rho_{\mathrm{n}} \boldsymbol{U}_{\mathrm{n}}}{e n_{\mathrm{i}}\left(m_{\mathrm{e}}+m_{\mathrm{i}}\right)}
\end{aligned}
$$

using the quasi-neutrality condition that $n_{\mathrm{e}}=n_{\mathrm{i}}$.

An approximate expression for the neutral fluid diffusion velocity in terms of $\boldsymbol{J}$ is (Mitchner \& Kruger 1973, Sect. IV.8)

$\boldsymbol{U}_{\mathrm{n}}=\frac{\rho_{\mathrm{i}}}{\rho}\left(-\frac{\rho_{\mathrm{n}}}{\rho n_{\mathrm{e}} m_{\mathrm{in}} \nu_{\mathrm{in}} c} \boldsymbol{J} \times \boldsymbol{B}-\left(\frac{m_{\mathrm{e}}}{m_{\mathrm{in}}}\right)^{1 / 2} \frac{\boldsymbol{J}}{e n_{\mathrm{e}}}\right)$.

Substitution of Eq. (44) into Eqs. (42) and (43) gives $\boldsymbol{U}_{\mathrm{e}}$ and $\boldsymbol{U}_{\mathrm{i}}$ in terms of $\boldsymbol{J}$ and $\boldsymbol{J} \times \boldsymbol{B}$. Since $\boldsymbol{J}_{\mathrm{e}}=-\boldsymbol{e} n_{\mathrm{e}} \boldsymbol{U}_{\mathrm{e}}$ and $\boldsymbol{J}_{\mathrm{i}}=\boldsymbol{e} \boldsymbol{n}_{\mathrm{i}} \boldsymbol{U}_{\mathrm{i}}$, this gives

$\boldsymbol{J}_{\mathrm{e}}=\left(1-\frac{\rho_{\mathrm{n}}}{\rho}\left(\frac{m_{\mathrm{e}}}{m_{\mathrm{in}}}\right)^{1 / 2}\right) \boldsymbol{J}-\frac{\Gamma}{M_{\mathrm{e}}} \boldsymbol{J} \times \hat{\boldsymbol{B}}$

and

$\boldsymbol{J}_{\mathrm{i}}=\frac{\rho_{\mathrm{n}}}{\rho}\left(\frac{m_{\mathrm{e}}}{m_{\mathrm{in}}}\right)^{1 / 2} \boldsymbol{J}+\frac{\Gamma}{M_{\mathrm{e}}} \boldsymbol{J} \times \hat{\boldsymbol{B}}$,

ignoring terms of order $m_{\mathrm{e}} / m_{\mathrm{i}}$. Equations (45) and (46) are transformed into equations for the elements of the electron and ion conductivity tensors in the following way.

Use the Ohm's law Eq. (4) for $\boldsymbol{J}$ to express the right hand sides of Eqs. (45) and (46) in terms of the elements of the total conductivity tensor. Next write the Ohm's laws for the electron and ion fluids as

$\boldsymbol{J}_{\mathrm{e}}=\sigma_{\|}^{(\mathrm{e})} \boldsymbol{E}_{\|}+\sigma_{\mathrm{P}}^{(\mathrm{e})} \boldsymbol{E}_{\perp}^{\prime}+\sigma_{\mathrm{H}}^{(\mathrm{e})} \boldsymbol{E}^{\prime} \times \hat{\boldsymbol{B}}$

$\boldsymbol{J}_{\mathrm{i}}=\sigma_{\|}^{(\mathrm{i})} \boldsymbol{E}_{\|}+\sigma_{\mathrm{P}}^{(\mathrm{i})} \boldsymbol{E}_{\perp}^{\prime}+\sigma_{\mathrm{H}}^{(\mathrm{i})} \boldsymbol{E}^{\prime} \times \hat{\boldsymbol{B}}$. 
Use Eqs. (47) and (48) to express the left hand sides of Eqs. (45) and (46) in terms of the elements of the electron and ion conductivity tensors. Then, each of Eqs. (45) and (46) becomes a linear combination of $\boldsymbol{E}_{\|}, \boldsymbol{E}_{\perp}^{\prime}$, and $\boldsymbol{E}^{\prime} \times \hat{\boldsymbol{B}}$ that is equal to zero. Since these components of $\boldsymbol{E}^{\prime}$ are orthogonal, the coefficient of each of these components must vanish in each equation. Setting these coefficients equal to zero gives the elements of the electron and ion conductivity tensors as

$$
\begin{aligned}
& \sigma_{\|}^{(\mathrm{e})}=\left(1-\frac{\rho_{\mathrm{n}}}{\rho}\left(\frac{m_{\mathrm{e}}}{m_{\mathrm{in}}}\right)^{1 / 2}\right) \sigma_{\|} \\
& \sigma_{\mathrm{P}}^{(\mathrm{e})}=\left(1-\frac{\rho_{\mathrm{n}}}{\rho}\left(\frac{m_{\mathrm{e}}}{m_{\mathrm{in}}}\right)^{1 / 2}\right) \sigma_{\mathrm{P}}+\frac{\Gamma}{M_{\mathrm{e}}} \sigma_{\mathrm{H}} \\
& \sigma_{\mathrm{H}}^{(\mathrm{e})}=-\frac{\Gamma}{M_{\mathrm{e}}} \sigma_{\mathrm{P}}+\left(1-\frac{\rho_{\mathrm{n}}}{\rho}\left(\frac{m_{\mathrm{e}}}{m_{\mathrm{in}}}\right)^{1 / 2}\right) \sigma_{\mathrm{H}}
\end{aligned}
$$

and

$$
\begin{aligned}
\sigma_{\|}^{(\mathrm{i})} & =\frac{\rho_{\mathrm{n}}}{\rho}\left(\frac{m_{\mathrm{e}}}{m_{\mathrm{in}}}\right)^{1 / 2} \sigma_{\|} \\
\sigma_{\mathrm{P}}^{(\mathrm{i})} & =\frac{\rho_{\mathrm{n}}}{\rho}\left(\frac{m_{\mathrm{e}}}{m_{\mathrm{in}}}\right)^{1 / 2} \sigma_{\mathrm{P}}-\frac{\Gamma}{M_{\mathrm{e}}} \sigma_{\mathrm{H}} \\
\sigma_{\mathrm{H}}^{(\mathrm{i})} & =\frac{\Gamma}{M_{\mathrm{e}}} \sigma_{\mathrm{P}}+\frac{\rho_{\mathrm{n}}}{\rho}\left(\frac{m_{\mathrm{e}}}{m_{\mathrm{in}}}\right)^{1 / 2} \sigma_{\mathrm{H}} .
\end{aligned}
$$

These equations satisfy $\sigma_{\|}=\sigma_{\|}^{(\mathrm{e})}+\sigma_{\|}^{(\mathrm{i})}, \sigma_{\mathrm{P}}=\sigma_{\mathrm{P}}^{(\mathrm{e})}+\sigma_{\mathrm{P}}^{(\mathrm{i})}$, and $\sigma_{\mathrm{H}}=\sigma_{\mathrm{H}}^{(\mathrm{e})}+\sigma_{\mathrm{H}}^{(\mathrm{i})}$, as required. However, Eqs. (49) and (52) are inconsistent with Eq. (14) which states that $\sigma_{\|}=\sigma_{\|}^{(\mathrm{e})}$. Equation (14) is an approximation which neglects the contribution of the ions to $\sigma_{\|}$. This is a good approximation, but it introduces a small error of order $\left(\rho_{\mathrm{n}} / \rho\right)\left(m_{\mathrm{e}} / m_{\mathrm{in}}\right)^{1 / 2} \leq$ $\left(m_{\mathrm{e}} / m_{\mathrm{in}}\right)^{1 / 2} \ll 1$ in the derivation of $\sigma_{\|}^{(\mathrm{e})}$ and $\sigma_{\|}^{(\mathrm{i})}$ from the total conductivities. Neglecting terms of this order compared to unity, and using the expressions for the total conductivities given by Eqs. (14)-(16), Eqs. (49)-(54) become

$\sigma_{\|}^{(\mathrm{e})}=\sigma_{\|}$

$\sigma_{\mathrm{P}}^{(\mathrm{e})}=\frac{\sigma_{\|}}{(1+\Gamma)^{2}+M_{\mathrm{e}}^{2}}$

$\sigma_{\mathrm{H}}^{(\mathrm{e})}=\frac{-\sigma_{\|}\left(\Gamma(1+\Gamma)+M_{\mathrm{e}}^{2}\right)}{M_{\mathrm{e}}\left((1+\Gamma)^{2}+M_{\mathrm{e}}^{2}\right)}$

$\sigma_{\|}^{(\mathrm{i})}=\frac{\rho_{\mathrm{n}}}{\rho}\left(\frac{m_{\mathrm{e}}}{m_{\mathrm{in}}}\right)^{1 / 2} \sigma_{\|}$

$\sigma_{\mathrm{P}}^{(\mathrm{i})}=\sigma_{\mathrm{P}}^{(\mathrm{e})}\left(\Gamma+\frac{\rho_{\mathrm{n}}}{\rho}\left(\frac{m_{\mathrm{e}}}{m_{\mathrm{in}}}\right)^{1 / 2}\right)$

$\sigma_{\mathrm{H}}^{(\mathrm{i})}=\sigma_{\mathrm{P}}^{(\mathrm{e})}\left(-\frac{\rho_{\mathrm{n}}}{\rho}\left(\frac{m_{\mathrm{e}}}{m_{\mathrm{in}}}\right)^{1 / 2} M_{\mathrm{e}}+\frac{\Gamma(1+\Gamma)}{M_{\mathrm{e}}}\right)$.

\subsection{Approximate conductivity tensor for the solar atmosphere}

The conductivities in Sect. 2.1 are for a three species plasma of electrons, singly charged ions, and neutrals. The number of species in the solar atmosphere is much larger than three. The atmosphere consists primarily of electrons, protons, singly charged metallic ions, H, He I, He II, and He III. Multiply charged ions other than HeIII exist in the transition region and corona, but their number density is orders of magnitude smaller than that of HeIII. For the purpose of computing an approximate conductivity tensor the atmosphere is modeled here as a three species plasma described in Sect. 2.2.1. Then the conductivities in Sect. 2.1 are used for the conductivities of this plasma. It is possible to extend the three species conductivity model in Mitchner \& Kruger (1973) to more species by straightforward but lengthy calculation. This is not done here since it is not expected to provide much more important information than the three species model.

\subsubsection{Model plasma composition}

The height dependent profiles of temperature and particle densities predicted by a model that is essentially model CM of Fontenla et al. (2002, henceforth FAL CM), as communicated by Avrett \& Loeser (2001), are used as inputs to the model. Although the FAL model used here differs slightly from FAL $\mathrm{CM}$, it is referred to here as FAL CM. The FAL models are extensions of models of Vernazza et al. (1981) above the Lyman $\alpha$ plateau. The FAL models are time and horizontally space averaged, hydrostatic, NLTE semi-empirical models. Although important dynamical features of the atmosphere cannot be described by these models (Carlsson \& Stein 2002), they provide a useful, quantitative first approximation to the vertical temperature and density structure of the chromosphere (Kalkofen 2001).

According to the FAL models, the vertical temperature and density structure of network, internetwork, and plage regions are similar. Assuming this is true, FAL CM is taken to describe all of these regions. The regions are distinguished here by different values of the photospheric magnetic field strength.

Let the densities of electrons, protons, H, HeI, HeII, HeIII, $\mathrm{C}, \mathrm{Si}, \mathrm{Al}, \mathrm{Mg}, \mathrm{Fe}, \mathrm{Na}$, and $\mathrm{Ca}$ be given by FAL CM. The $\mathrm{H}$ density includes atomic and molecular hydrogen, and the HeI density includes excited HeI.

Let the singly charged metallic ions be collectively represented by a single proxy metallic ion (henceforth proxy ion), denoted by i. The metallic ions contribute most of the free electrons, and emit most of the net radiative loss in the lower chromosphere (Anderson \& Athay 1989 a,b).

The proxy ion number density $n_{\mathrm{i}}$ is determined by the quasi-neutrality condition

$n_{\mathrm{i}}=n_{\mathrm{e}}-\left(n_{\mathrm{p}}+n_{\mathrm{HeII}}+2 n_{\mathrm{HeIII}}\right)$,

which states that the total charge density is zero in the MHD approximation.

The proxy ion mass $m_{\mathrm{i}}(z)$ is defined by

$m_{\mathrm{i}}(z)=\frac{\rho_{\mathrm{C}}+\rho_{\mathrm{Si}}+\rho_{\mathrm{Al}}+\rho_{\mathrm{Mg}}+\rho_{\mathrm{Fe}}+\rho_{\mathrm{Na}}+\rho_{\mathrm{Ca}}}{n_{\mathrm{C}}+n_{\mathrm{Si}}+n_{\mathrm{Al}}+n_{\mathrm{Mg}}+n_{\mathrm{Fe}}+n_{\mathrm{Na}}+n_{\mathrm{Ca}}}$,

where $z$ is the height above the base of the photosphere at $z=0$. Here the photosphere is defined to be the region between $z=0$ 
and the height $H_{T \min }=525 \mathrm{~km}$ of the FAL CM temperature minimum.

The protons and proxy ions are collectively represented by an effective singly charged ion denoted by i, eff. Mathematically, the effective ion enters the model only through its magnetization $M_{\mathrm{i}, \text { eff }}$ and its mass $m_{\mathrm{i}, \text { eff }}$. They are defined by

$$
\begin{aligned}
M_{\mathrm{i}, \text { eff }} & =\frac{n_{\mathrm{i}} M_{\mathrm{i}}+n_{\mathrm{p}} M_{\mathrm{p}}}{n_{\mathrm{i}}+n_{\mathrm{p}}} \\
m_{\mathrm{i}, \text { eff }} & =\frac{n_{\mathrm{i}} m_{\mathrm{i}}+n_{\mathrm{p}} m_{\mathrm{p}}}{n_{\mathrm{i}}+n_{\mathrm{p}}} .
\end{aligned}
$$

He II and He III are omitted from the definition of i, eff. The reason is that their number densities are many orders of magnitude smaller than those of proxy ions and protons in the photosphere and chromosphere so that their relative contribution to the current density in this region is insignificant. In the transition and corona, $n_{\mathrm{e}} \sim n_{\mathrm{p}} \gg n_{\text {HeIII }} \gg n_{\mathrm{i}} \gg n_{\text {HeII }}$, but there the current density is almost entirely carried by electrons.

As discussed in Sect. 4, and shown in Fig. 6, the reduced collision frequencies of protons and proxy ions with one another are orders of magnitude less than their reduced collision frequencies with $\mathrm{H}$ and $\mathrm{HeI}$ from the photosphere to the upper chromosphere. Then the protons and proxy ions scatter independently of one another so to a good approximation their contributions to the collisional rate of momentum transfer to the effective ion are additive. Collisions of protons and proxy ions with electrons, HeII and HeIII are not important due to the relatively small mass of the electrons and the small number densities of the He ions compared with the number densities of protons and $\mathrm{H}$.

$\mathrm{H}$ and $\mathrm{HeI}$ are collectively represented by a single neutral component with a mass

$m_{\mathrm{n}}=\frac{n_{\mathrm{H}} m_{\mathrm{p}}+n_{\mathrm{HeI}} m_{\mathrm{HeI}}}{n_{\mathrm{H}}+n_{\mathrm{HeI}}}$.

This procedure of representing several particle species by a single proxy particle species allows the plasma to be approximately described by a three species plasma. The model plasma then consists of electrons, a singly charged ion species that represents protons and metallic ions, and a neutral species that represents $\mathrm{H}$ and $\mathrm{HeI}$.

\subsubsection{Collision frequencies and magnetizations}

The general definitions of the magnetization and reduced collision frequencies for any species $s$ are given by Eqs. (25)-(28).

The magnetizations of electrons, protons, and proxy ions are

$$
\begin{aligned}
& M_{\mathrm{e}}=\frac{\omega_{\mathrm{e}}}{v_{\mathrm{e}}^{*}} \\
& M_{\mathrm{p}}=\frac{\omega_{\mathrm{p}}}{v_{\mathrm{p}}^{*}} \\
& M_{\mathrm{i}}=\frac{\omega_{\mathrm{i}}}{v_{\mathrm{i}}^{*}},
\end{aligned}
$$

where the total reduced collision frequencies are computed from

$v_{\mathrm{e}}^{*}=v_{\mathrm{ep}}^{*}+v_{\mathrm{ei}}^{*}+v_{\mathrm{eH}}^{*}+v_{\mathrm{e}, \mathrm{HeI}}^{*}+v_{\mathrm{e}, \mathrm{HeII}}^{*}+v_{\mathrm{e}, \mathrm{HeIII}}^{*}$

$$
\begin{aligned}
& v_{\mathrm{p}}^{*}=v_{\mathrm{pe}}^{*}+v_{\mathrm{pi}}^{*}+v_{\mathrm{pH}}^{*}+v_{\mathrm{p}, \text { HeI }}^{*}+v_{\mathrm{p}, \text { HeII }}^{*}+v_{\mathrm{p}, \text { HeIII }}^{*} \\
& v_{\mathrm{i}}^{*}=v_{\mathrm{ie}}^{*}+v_{\mathrm{ip}}^{*}+v_{\mathrm{iH}}^{*}+v_{\mathrm{i}, \text { HeI }}^{*}+v_{\mathrm{i}, \text { HeII }}^{*}+v_{\mathrm{i}, \text { HeIII }}^{*}
\end{aligned}
$$

The collision frequencies $v_{k l}$ that are needed to compute $v_{k l}^{*}$ are given by standard expressions listed in Sect. 2.2.3. They are functions of temperature, and of the particle number densities and masses.

\subsubsection{Collision frequencies}

The collision frequencies that enter into the computation of the electrical conductivity are of two types: charged-neutral and charged-charged. Let the subscripts $0,+$, and e denote neutral particles, positively charged particles, and electrons. Let the subscript $+j$ denote positively charged particles of type $j$. Let $\sigma=5 \times 10^{-15} \mathrm{~cm}^{2}$ be the scattering cross section for charged-neutral scattering (Krall \& Trivelpiece 1986; Book 1994). Let $m_{i j}$ be the reduced mass of particles $i$ and $j$.

The charged-neutral collision frequency is

$v_{\text {or } \mathrm{e}, 0}=n_{0} \sigma\left(\frac{3 k_{\mathrm{B}} T}{m_{+ \text {or } \mathrm{e}, 0}}\right)^{1 / 2}$.

There are three types of charged-charged collision frequencies.

$\nu_{+, \mathrm{e}}=\frac{4 \sqrt{2 \pi}}{3} \frac{Z_{+}^{2} e^{4} n_{\mathrm{e}} \ln \Lambda}{m_{\mathrm{e}}^{1 / 2}\left(k_{\mathrm{B}} T\right)^{3 / 2}}$

where $Z_{+}$is the charge number of the positively charged particle.

$v_{\mathrm{e},+}=\frac{4 \sqrt{2 \pi}}{3} \frac{Z_{+}^{2} e^{4} n_{+} \ln \Lambda}{m_{\mathrm{e}}^{1 / 2}\left(k_{\mathrm{B}} T\right)^{3 / 2}}$.

$\nu_{+1,+2}=\frac{4 \sqrt{\pi}}{3} \frac{Z_{1+}^{2} Z_{2+}^{2} e^{4} n_{+2} \ln \Lambda}{m_{1+, 2+}^{1 / 2}\left(k_{\mathrm{B}} T\right)^{3 / 2}}$.

Here $\ln \Lambda$ is the Coulomb logarithm, defined by

$\ln \Lambda=\ln \left(\frac{3 \lambda_{D} k_{\mathrm{B}} T}{Z_{1} Z_{2} e^{2}}\right)$

where $Z_{1}$ and $Z_{2}$ are the charge numbers of the two colliding particles, and $\lambda_{\mathrm{D}}$ is the Debye length for a multi-species plasma, defined by (Ichimaru 1973)

$\lambda_{\mathrm{D}}=\left(\sum_{s} \frac{4 \pi n_{s} q_{s}^{2}}{k_{\mathrm{B}} T}\right)^{-1 / 2}$,

where the sum is over all charged species.

\subsubsection{Conductivities}

The elements of the total conductivity tensor are finally given by Eqs. (14)-(18) with the ion species being the effective ion species that represents protons and metallic ions, and the neutral species being the proxy neutral species that represents $\mathrm{H}$ and HeI, as defined in Sect. 2.1.1. The elements of the total 
conductivity tensor for this three species representation of the solar atmospheric plasma are then

$$
\begin{aligned}
\sigma_{\|} & =\sigma_{\|}^{(\mathrm{e})} \\
\sigma_{\mathrm{P}} & =\frac{\sigma_{\|}(1+\Gamma)}{(1+\Gamma)^{2}+M_{\mathrm{e}}^{2}} \\
\sigma_{\mathrm{H}} & =\frac{-M_{\mathrm{e}} \sigma_{\|}}{(1+\Gamma)^{2}+M_{\mathrm{e}}^{2}} \\
\sigma_{\|}^{(\mathrm{e})} & =\frac{n_{\mathrm{e}} e^{2}}{m_{\mathrm{e}} v_{\mathrm{e}}^{*}} \\
\Gamma & =\left(\frac{\rho_{\mathrm{n}}}{\rho}\right)^{2} M_{\mathrm{e}} M_{\mathrm{i}, \mathrm{eff}} .
\end{aligned}
$$

$M_{\mathrm{e}}$ is given by Eq. (66). $M_{\mathrm{i}, \text { eff }}$ is given by Eqs. (63), (67), and (68).

$$
\begin{aligned}
\rho & =\rho_{\mathrm{e}}+\rho_{\mathrm{p}}+\rho_{\mathrm{i}}+\rho_{\mathrm{H}}+\rho_{\mathrm{HeI}}+\rho_{\mathrm{HeII}}+\rho_{\mathrm{HeIII}} \\
\rho_{\mathrm{n}} & =\rho_{\mathrm{H}}+\rho_{\mathrm{HeI}} .
\end{aligned}
$$

Similarly, the elements of the electron and effective ion conductivity tensors are formally obtained from Eqs. (55)-(60) by letting $\mathrm{i} \rightarrow \mathrm{i}$, eff, and using Eqs. (64) and (65) to compute $m_{(\mathrm{i}, \text { eff)n }}$. The conductivity tensors, and $Q$ as given by Eq. (34) may then be computed once $B$ is known.

\section{Magnetic field magnitude}

Only the magnitude of the magnetic field appears in the model. It is assumed to be given by

$$
B(z)=B(0) \exp \left(-\gamma \int_{0}^{z} \frac{\mathrm{d} \eta}{2 L(\eta)}\right)
$$

Here

$$
L(z)=\frac{k_{\mathrm{B}} T(z)}{\langle m(z)\rangle g}
$$

is the ideal gas pressure scale height for a multi-species plasma, where the average particle mass $\langle m(z)\rangle$ is defined by

$\langle m(z)\rangle=\frac{\rho}{n}$.

Here $n$ is the total number density, $g=2.74 \times 10^{4} \mathrm{~cm} \mathrm{~s}^{-2}$ is the solar gravitational acceleration at the photosphere, $k_{\mathrm{B}}$ is Boltzmann's constant, and $\gamma=0.7326$ is an ad hoc constant chosen so that $B(z)$ decreases by a factor of 100 from the photosphere to the maximum height $z_{\max } \sim 1.904 \times 10^{4} \mathrm{~km}$ of the FAL CM atmosphere. Since $T\left(z_{\max }\right)=1.58631 \times 10^{6} \mathrm{~K}, \mathrm{FAL}$ $\mathrm{CM}$ includes the lower corona.

Any reasonable choice for the variation of $B$ with height gives the same basic numerical results reported in Sect. 4. The reason is that these results follow primarily from the rapid increase of particle magnetization with height that begins near $H_{T \min }$. When the plasma is weakly ionized, only charged particle-H collisions are important in determining the conductivity, in which case $M_{s} \propto B T^{1 / 2} / n_{\mathrm{H}}$. When the plasma is mostly ionized it is primarily electrons and protons, and electron-proton collisions that determine the conductivity with $n_{\mathrm{e}} \sim n_{\mathrm{p}}$, in which case $M_{s} \propto B T^{3 / 2} / n_{s}$ for $s=\mathrm{e}, \mathrm{p}$. Then, largely independent of the degree of ionization, the rapid increase in temperature, and rapid decrease in density with increasing height ensure that $M_{s}$ rapidly increases with height near $H_{T \text { min }}$ from values $\lesssim 1$ to values $\gg 1$ for any realistic variation of $B$ with height.

The analysis in this paper is independent of the geometry of the magnetic field since the conductivities depend on the magnetic field only through its magnitude. The analysis in this paper is also independent of any assumption about the mechanism that generates the electric field that drives the Pedersen current. Then the result shown in Figs. 4 and 10 that $Q$ rapidly approaches unity in the lower chromosphere, remains unity into the upper chromosphere, and then rapidly decreases to very small values in the transition region and lower corona is valid for any mechanism that generates a $\mathrm{CM}$ electric field that drives current orthogonal to the magnetic field. Similarly, the proof in Sect. 5 that for any weakly ionized plasma $Q$ rapidly approaches unity as the charged particles become magnetized is valid for any such mechanism.

\section{Numerical examples and general properties of solutions}

The numerical examples are generated using the conductivities in Sect. 2.2.4. The only input to the model that remains to be specified is $B(0)$, the magnetic field strength at the base of the photosphere. Figures 1-9 show properties of the solution to the model for $B(0)=500 \mathrm{G}$. The height range for all figures is from $z=0$ to $z=1.904 \times 10^{4} \mathrm{~km}$. In all but one of these figures a logarithmic height scale is used, and the height range is shifted upward by $1 \mathrm{~km}$ to ensure that $z=0$ corresponds to $\log _{10}(z+1)=0$.

Figures 1 and 2 show the profiles of temperature, magnetic field strength, proxy ion mass, and number densities that are inputs to the model. $H_{T \min }=525 \mathrm{~km}$ where $T=4500 \mathrm{~K}$.

Figures 3 and 4 show the total parallel, Pedersen, and Hall conductivities, the efficiency $Q$ of Pedersen current dissipation, and the magnetization of the charged particles as functions of height. The ratio $\sigma_{\mathrm{P}} / \sigma_{\mathrm{H}}$ determines the heating efficiency $Q$ through Eq. (34), and the magnetizations $M_{\mathrm{e}}$ and $M_{\mathrm{i}, \text { eff }}$ play a major role in determining $\sigma_{\mathrm{P}}$ and $\sigma_{\mathrm{H}}$.

From the photosphere to the upper chromosphere, $\rho_{\mathrm{n}} / \rho \sim$ 1. This height range is divided into three regions in Figs. 3 and 4 .

The first region is near $z=0$. In this region $M_{\mathrm{e}} \ll 1$. This implies that $M_{\mathrm{i}, \text { eff }} \ll 1$ since the proton and metallic ion masses are much larger than the electron mass, and since the magnetization of a species $s$ is roughly $\propto m_{s}^{-1 / 2}$. Then from Eqs. (34), (79), and (80) it follows that $Q \sim 1$ and $\sigma_{\mathrm{P}} \sim \sigma_{\|} \gg \sigma_{\mathrm{H}}$. Although $Q \sim 1$ in this region there is no essential difference between Pedersen current dissipation and magnetic field aligned (parallel) current dissipation. The reason is that the magnetizations are so small that the conductivity tensor is almost isotropic, so the conductivity in any direction is essentially $\sigma_{\|}$. The heating rate in this region is $\sim \sigma_{\|}\left|\boldsymbol{E}^{\prime}\right|^{2}$, and the current in any direction is almost entirely an electron current. Although $\boldsymbol{B}$ does not have a significant effect on the 


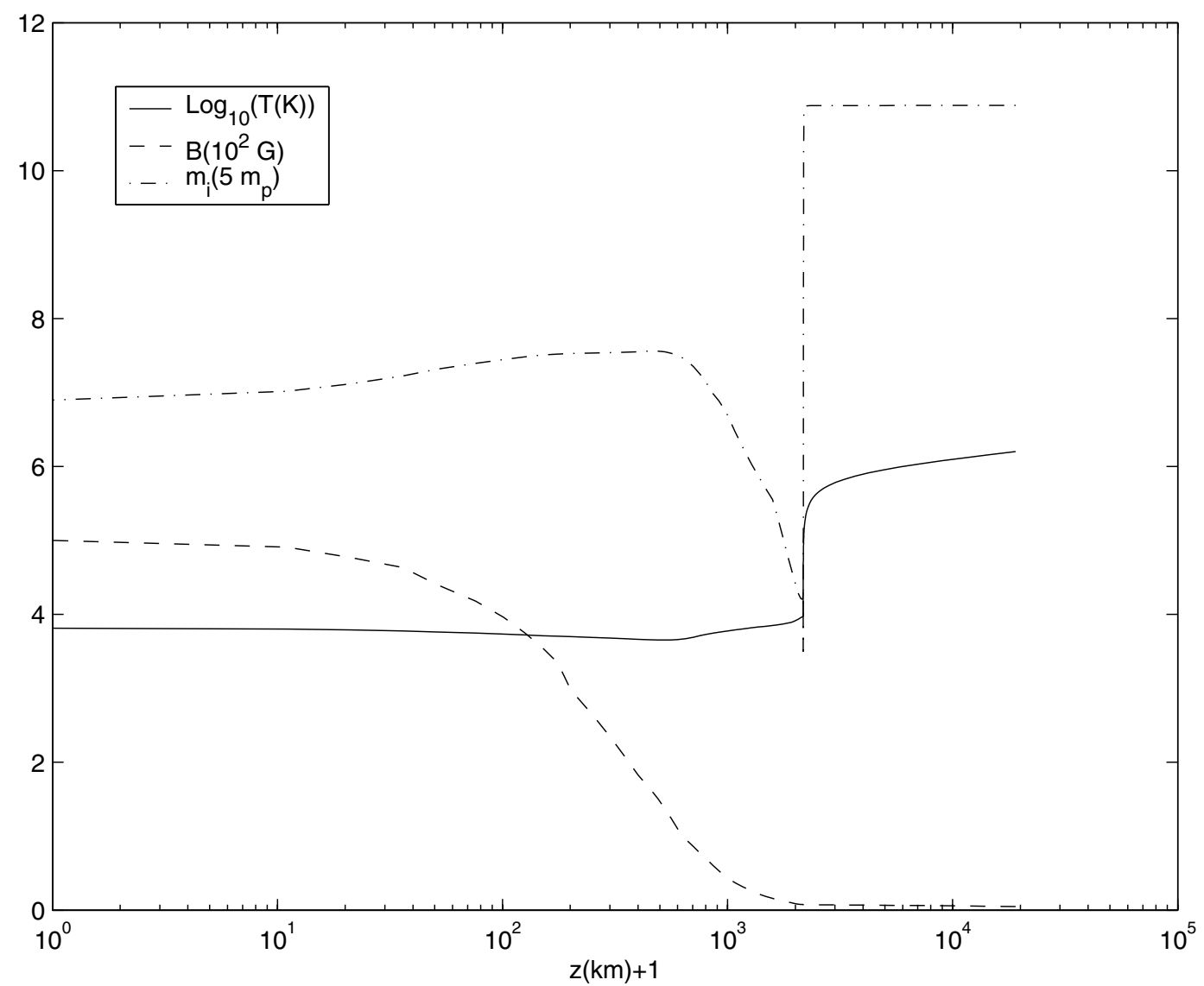

Fig. 1. Temperature, magnetic field strength, and proxy ion mass as functions of height. The proxy ion mass is given in units of five times the proton mass.

conductivity tensor, it can play an important role in determining the heating rate since it partly determines $\boldsymbol{E}_{\perp}^{\prime}$.

A necessary and sufficient condition that Pedersen current dissipation be both efficient and significantly different from parallel current dissipation is that $\sigma_{\mathrm{H}} \ll \sigma_{\mathrm{P}} \ll \sigma_{\|}$. The first inequality states that $Q \sim 1$. The second inequality is based on the fact that $\sigma_{\mathrm{P}} \leq \sigma_{\|}$, with equality holding when $B=0$. In general, in the photosphere and chromosphere,

$$
Q \sim \frac{1}{1+\frac{M_{\mathrm{e}}^{2}}{\left(1+M_{\mathrm{e}} M_{\mathrm{i}, \mathrm{eff}}\right)^{2}}},
$$

and

$$
\frac{\sigma_{\mathrm{P}}}{\sigma_{\|}} \sim \frac{1+M_{\mathrm{e}} M_{\mathrm{i}, \mathrm{eff}}}{\left(1+M_{\mathrm{e}} M_{\mathrm{i}, \mathrm{eff}}\right)^{2}+M_{\mathrm{e}}^{2}} .
$$

It follows from Eq. (88) that $Q \sim 1 \Leftrightarrow M_{\mathrm{e}} \ll 1$ or $M_{\mathrm{i} \text {, eff }} \gtrsim 2$, and from Eq. (89) that $\sigma_{\mathrm{P}} / \sigma_{\|} \ll 1 \Leftrightarrow M_{\mathrm{e}} \gg 1$, using $M_{\mathrm{e}} \gg$ $M_{\mathrm{i}, \text { eff }}$. Then Pedersen current dissipation can be important only in regions where $M_{\mathrm{e}} \gg 1$ and $M_{\mathrm{i} \text {, eff }} \gtrsim 2$. As discussed in the context of Figs. 10 and 11, the range $100 \leq B(0) \leq 1500 \mathrm{G}$ defines a transition layer in the height range $\Delta H \sim 400-800 \mathrm{~km}$ that separates underlying plasma in which Pedersen current dissipation is not important from overlying plasma in which $M_{\mathrm{e}} \gg 1$ and $M_{\mathrm{i}, \text { eff }} \gtrsim 2$, and hence in which Pedersen current dissipation is a distinctly different heating mechanism from parallel current dissipation, and is very efficient, and so can be important.

The second region extends from $z \sim 100-200 \mathrm{~km}$ to $H_{T \text { min }}$. In this region $M_{\mathrm{e}} M_{\mathrm{i} \text {, eff }} \lesssim 1, Q \sim\left(1+M_{\mathrm{e}}^{2}\right)^{-1}$, and $\sigma_{\mathrm{P}} \lesssim \sigma_{\mathrm{H}} \ll \sigma_{\|} . Q$ rapidly decreases with increasing height since $M_{\mathrm{e}}$ rapidly increases with height. The minimum value of $Q$ is 0.09 , and occurs at $H_{T \text { min }}$. In this region the conductivity tensor is anisotropic, but heating by Pedersen current dissipation is inefficient.

The third region is the chromosphere. In this region $M_{\mathrm{i} \text {, eff }} \gg 1$ so that $Q \sim 1 /\left(1+M_{\mathrm{i} \text {, eff }}^{-2}\right) \sim 1$, and $\sigma_{\mathrm{H}} \ll \sigma_{\mathrm{P}} \ll \sigma_{\|}$. There is a rapid transition between the second and third regions caused by the rapid increase of $M_{\mathrm{e}}$ and $M_{\mathrm{i}}$, eff with height in the lower chromosphere. Over the height range from $H_{T \text { min }}$ to $z=1036 \mathrm{~km}, Q$ increases by two orders of magnitude from 0.09 to 0.9 . This is the height range of the lower chromosphere. $Q$ first reaches 0.5 at $z=813 \mathrm{~km}$, which is the height at which $M_{\mathrm{p}}$ reaches unity. $M_{\mathrm{i}}$ first reaches unity at $z=881 \mathrm{~km}$. There is a strong positive correlation between the rapid increase of $Q$ from $\ll 1$ to $\sim 1$, and the rapid increase of $M_{\mathrm{i} \text {, eff }}$ from $\ll 1$ to $\gg 1$ in the lower chromosphere. This rapid increase of $M_{\mathrm{i} \text {, eff }}$ causes $\boldsymbol{J}_{\perp}$ to be rapidly transformed from being orthogonal to $\boldsymbol{E}_{\perp}^{\prime}$, and hence a Hall current density, to being parallel to $\boldsymbol{E}_{\perp}^{\prime}$, and hence a Pedersen current density, thereby maximizing the efficiency $Q$ with which the CM electric field transfers energy to the plasma that is thermalized by collisions. From $z=1036 \mathrm{~km}$, 


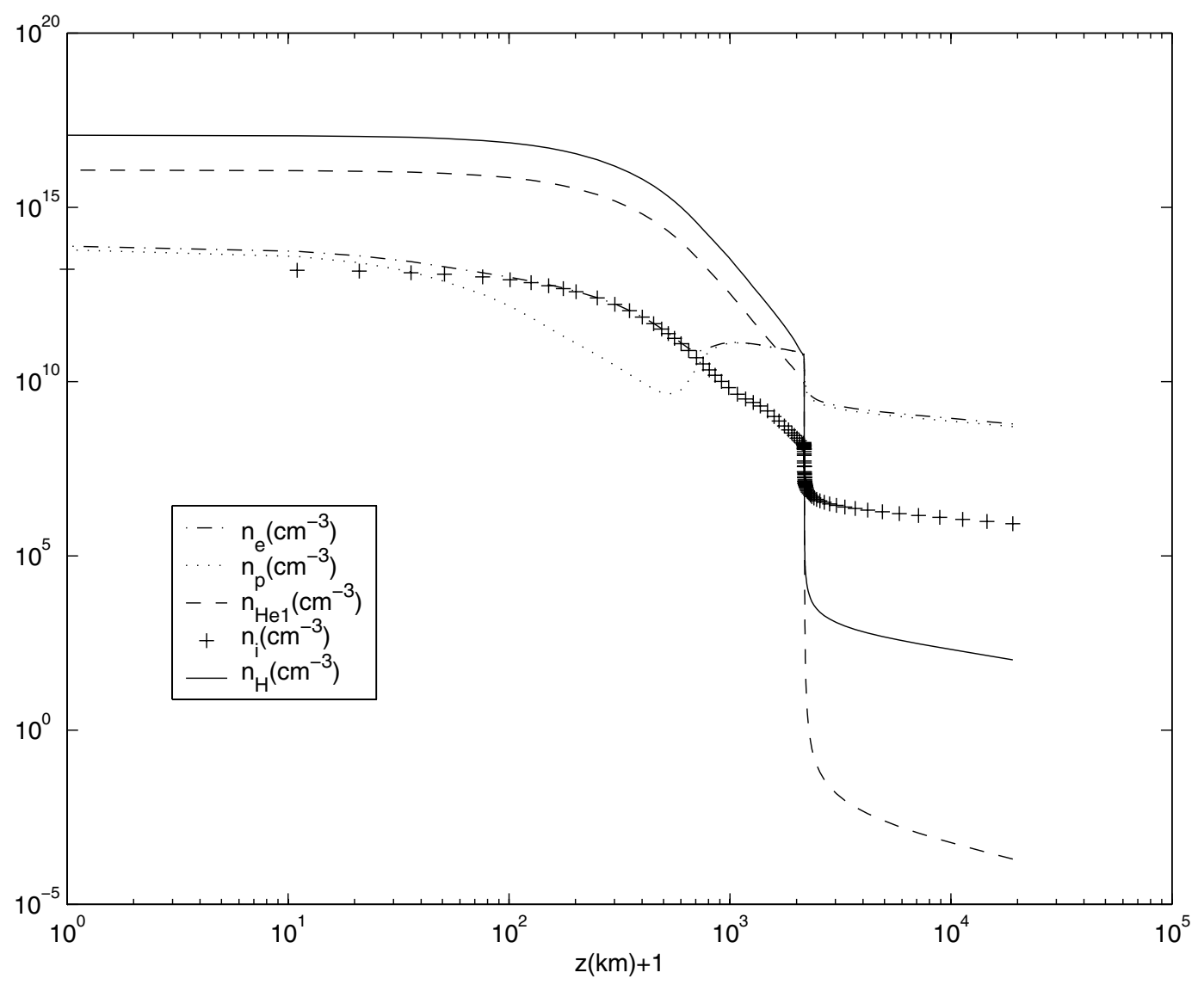

Fig. 2. Number densities of H, HeI, electrons, protons, and proxy ions as functions of height. The densities of HeII and HeIII are not shown since they are orders of magnitude less than the plotted number densities in the photosphere and chromosphere, and do not make a significant contribution to the Pedersen current anywhere.

$Q$ rapidly increases from 0.9 to unity, remains equal to unity into the upper chromosphere up to $z \sim 2168 \mathrm{~km}$, and then, as the plasma rapidly becomes highly ionized, decreases precipitously to very small values in the transition region and lower corona. Then current that flows perpendicular to the magnetic field is dissipated with high efficiency in the chromosphere, but is dissipated with very low efficiency in the overlying highly ionized atmosphere. Even for $B(0)=1500 \mathrm{G}$ the range of $Q$ from the transition region into the lower corona is from $\sim 10^{-5}$ at $z=2170 \mathrm{~km}$ to $\sim 10^{-14}$ at $z=1.9 \times 10^{4} \mathrm{~km}$.

The reason $Q \rightarrow 0$ as the plasma becomes highly ionized is as follows. For $B(0) \lesssim 3000 \mathrm{G}$ the rapid decrease of $\rho_{\text {n }} / \rho$ with height to small values beginning in the transition region causes $\Gamma$ to decrease to $\ll 1$. Then $Q \sim M_{\mathrm{e}}^{-2} \ll 1$ since $M_{\mathrm{e}} \gg 1 . Q \ll 1$ in any fully ionized plasma when the electrons are strongly magnetized. Heating by Pedersen current dissipation is very inefficient in a fully ionized, strongly magnetized plasma.

The rapid decrease of $Q$ to very small values in the transition region and corona is consistent with the model result in Goodman (1998) that the resistive dissipation rate of currents in the transition region on the spatial scales of the FAL models is many orders of magnitude smaller than the net radiative loss from this region. These results suggest that Pedersen current dissipation is not significant for heating the transition region or corona on the spatial scales of the FAL models.

Figure 5 shows the ratio $J_{\mathrm{P}}^{(\mathrm{i}, \mathrm{eff})} / J_{\mathrm{P}}^{(\mathrm{e})}$ as a function of height. The Pedersen current is an electron current for $z<H_{T \min }$, rapidly makes the transition to an effective ion current above $H_{T \text { min }}$, remains an effective ion current throughout the chromosphere, and rapidly changes back to an electron current in the transition region. In general,

$$
\begin{aligned}
\frac{J_{\mathrm{P}}^{(\mathrm{i}, \text { eff })}}{J_{\mathrm{P}}^{(\mathrm{e})}} & =\frac{\sigma_{\mathrm{P}}^{(\mathrm{i}, \text { eff })}}{\sigma_{\mathrm{P}}^{(\mathrm{e})}} \\
& =\frac{\rho_{\mathrm{n}}}{\rho}\left(\left(\frac{m_{\mathrm{e}}}{m_{\mathrm{i}, \text { eff }) \mathrm{n}}}\right)^{1 / 2}+\frac{\rho_{\mathrm{n}}}{\rho} M_{\mathrm{e}} M_{\mathrm{i}, \text { eff }}\right) .
\end{aligned}
$$

In the photosphere and chromosphere, where $\rho_{\mathrm{n}} / \rho \sim 1$, the right hand side of Eq. (91) is $\ll 1$ for $M_{\mathrm{e}} M_{\mathrm{i} \text {, eff }} \ll 1$, and is $\sim M_{\mathrm{e}} M_{\mathrm{i}, \text { eff }}$ for $M_{\mathrm{e}} M_{\mathrm{i}, \text { eff }} \gg\left(m_{\mathrm{e}} / m_{(\mathrm{i}, \text { eff }) \mathrm{n}}\right)^{1 / 2}$. The rapid increase of $M_{\mathrm{e}} M_{\mathrm{i}}$, eff near $H_{T \text { min }}$ from $\ll 1$ to $\gg 1$ with increasing height in the lower chromosphere implies that the effective ions carry the Pedersen current in the chromosphere. In and above the transition region, $\rho_{\mathrm{n}} / \rho \rightarrow 0$ in which case $J_{\mathrm{P}}^{(\mathrm{i}, \text { eff })} / J_{\mathrm{P}}^{(\mathrm{e})} \sim \Gamma$. For $B(0)=1500 \mathrm{G}, \Gamma$ decreases to 0.1372 in the corona. For other values of $B(0), \Gamma$ decreases to $0.1372(B(0)(\mathrm{G}) / 1500)^{2}$. The electrons dominate the Pedersen current in the corona except for $B(0) \gtrsim 3000 \mathrm{G}$. 


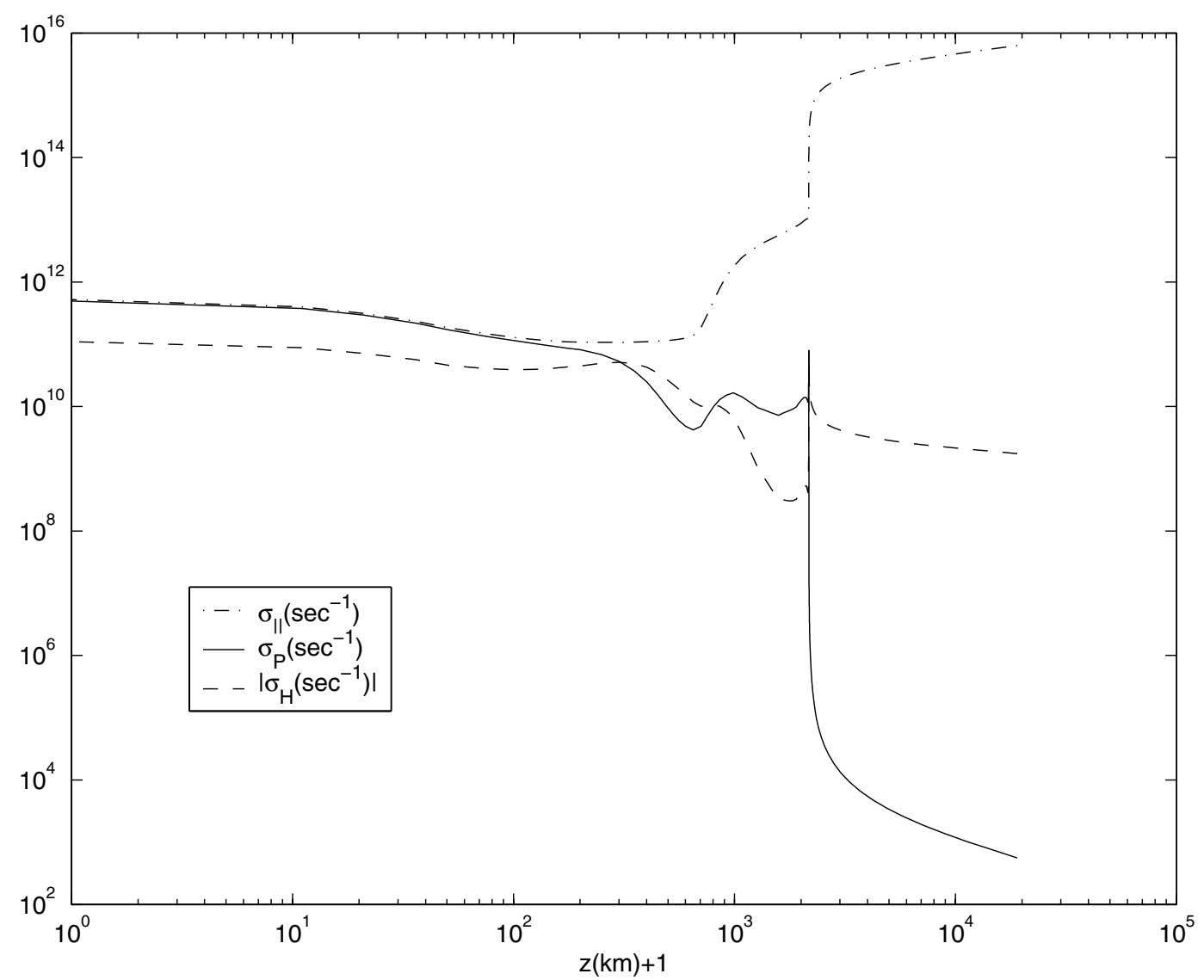

Fig. 3. Total parallel, Pedersen, and Hall electrical conductivities as functions of height.

Figure 6 shows the relative importance of electron-neutral versus electron-proxy ion and electron-proton collisions, of proton-neutral versus proton-proxy ion collisions, and of proxy ion-neutral versus proxy ion-proton collisions. Collisions of electrons with HeII and HeIII, and of protons and proxy ions with HeII, HeIII, and electrons are relatively unimportant. Figure 6 shows that from the photosphere to the upper chromosphere the charged-neutral reduced collision frequencies of protons and proxy ions are much greater than their chargedcharged reduced collision frequencies. This means that in this height range the conductivities of the protons and proxy ions are those for a weakly ionized plasma. This allows for an accurate estimate of the relative contribution of the protons and proxy ions to the effective ion Pedersen current in the chromosphere. This estimate is obtained as follows. The conductivity tensors in a weakly ionized plasma are given in Sect. 5. In particular, the Pedersen conductivity is given by Eq. (98). Then

$$
\begin{aligned}
\frac{J_{\mathrm{P}}^{(\mathrm{p})}}{J_{\mathrm{P}}^{(\mathrm{i})}} & =\frac{\sigma_{\mathrm{P}}^{(\mathrm{p})}}{\sigma_{\mathrm{P}}^{(\mathrm{i})}} \\
& =\frac{n_{\mathrm{p}}}{n_{\mathrm{i}}}\left(\frac{m_{\mathrm{i}}\left(m_{\mathrm{i}}+m_{\mathrm{n}}\right)}{m_{\mathrm{p}}\left(m_{\mathrm{p}}+m_{\mathrm{n}}\right)}\right)^{1 / 2} \frac{1+M_{\mathrm{i}}^{2}}{1+M_{\mathrm{p}}^{2}}
\end{aligned}
$$

from the photosphere to the upper chromosphere. Figure 7 shows a plot of $J_{\mathrm{P}}^{(\mathrm{p})} / J_{\mathrm{P}}^{(\mathrm{i})}$ as given by Eq. (93) for $0 \leq z \leq$ $2157 \mathrm{~km}$. The figure shows that $J_{\mathrm{P}}^{(\mathrm{p})}=J_{\mathrm{P}}^{(\mathrm{i})}$ at $z=185 \mathrm{~km}$, and at $z=589 \mathrm{~km}$, just above $H_{T \min }$ where the proton density begins to rapidly increase from its minimum value, and where, according to Fig. $2, n_{\mathrm{i}} / n_{\mathrm{p}}$ is a maximum. At $z=589 \mathrm{~km}, Q$ is essentially at its minimum value of $\sim 0.09$, and rapidly increases to unity above this height. The Pedersen current throughout the region of the chromosphere where $Q \sim 1$ is a proton current.

Figure 8 shows the fractional contribution of the electrons and effective ions to the total Hall current as a function of height. The Hall current is an electron current everywhere except for $z \sim 1000-2170 \mathrm{~km}$ where the electron and effective ion Hall conductivities, and hence currents, largely cancel. This cancelation begins to occur rapidly at $z \sim 700 \mathrm{~km}$, and leads to small values of $\left(\sigma_{\mathrm{H}} / \sigma_{\mathrm{P}}\right)^{2}$, and hence to values of $Q \sim 1$. The electron and effective ion Hall currents have opposite sign in the height range $0 \leq z \leq 2170 \mathrm{~km}$, which is the photosphere and chromosphere, and otherwise have the same sign.

Figure 9 shows the ratio of the magnitudes of the Pedersen and Hall current densities for the electrons and effective ions as a function of height. The Pedersen current dominates in regions where the magnetization is small, but, as already discussed, in such regions the conductivity tensor is approximately isotropic, and there is no essential difference between Pedersen and parallel current dissipation. In the chromosphere the Hall currents dominate due to the large magnetizations. Although the electron and effective ion Hall currents dominate the associated Pedersen currents throughout most of the chromosphere, they largely cancel in this region as discussed in the context of Fig. 8. The behavior in the transition region and corona is 


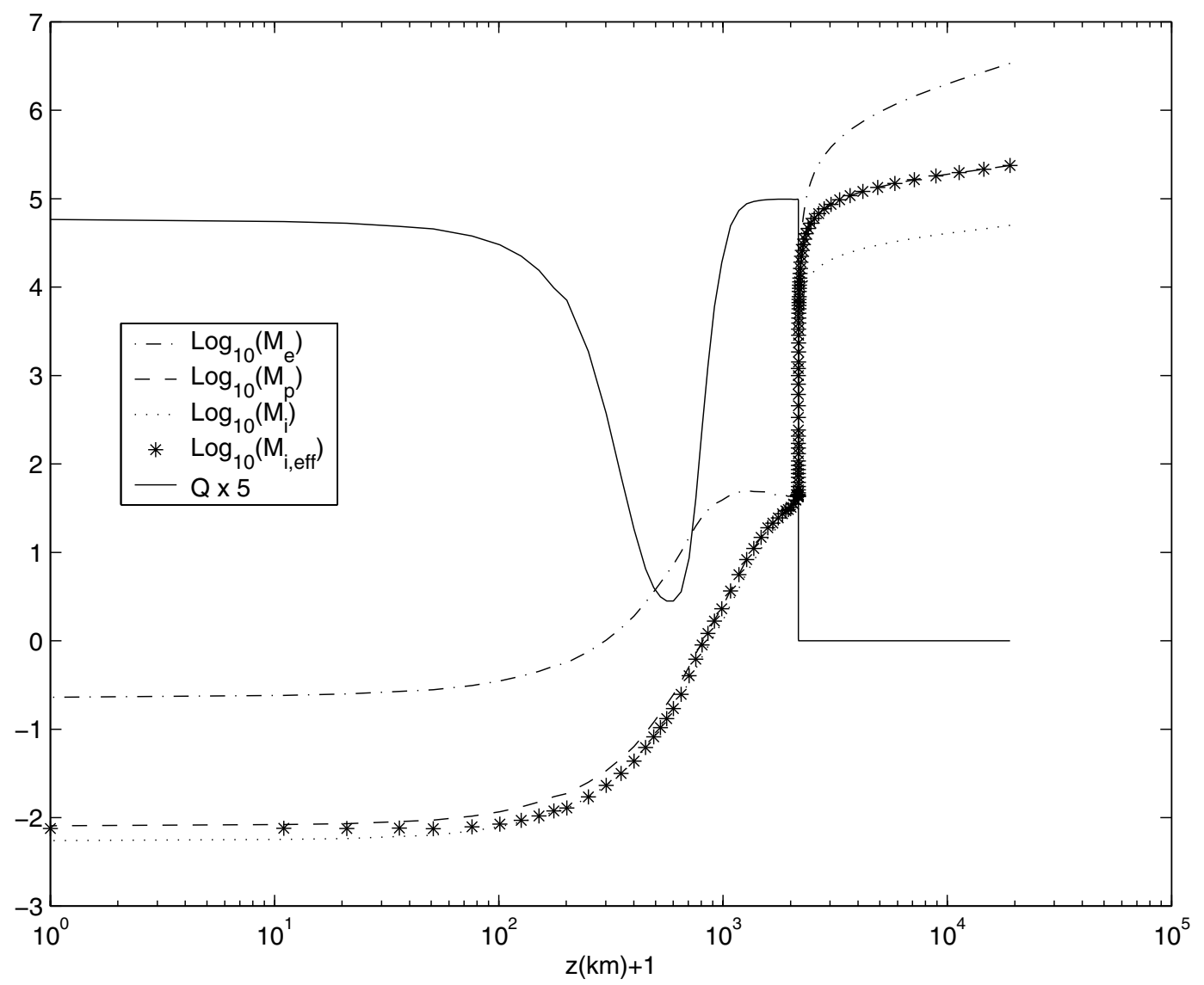

Fig. 4. Heating efficiency, and magnetization of electrons, protons, proxy ions, and effective ions as functions of height.

complicated by the rapid decrease of $\rho_{\mathrm{n}} / \rho$ from $\sim 1$ below the transition region to $\ll 1$ in and above the transition region. The general behavior of the ratio for the electrons may be understood analytically as follows. Since $M_{\mathrm{e}} \gg\left(\rho_{\mathrm{n}} / \rho\right)^{2} M_{\mathrm{i}, \text { eff }}$, Eqs. (56) and (57) imply that

$$
\begin{aligned}
\left|J_{\mathrm{P}}^{(\mathrm{e})} / J_{\mathrm{H}}^{(\mathrm{e})}\right| & =\left|\sigma_{\mathrm{P}}^{(\mathrm{e})} / \sigma_{\mathrm{H}}^{(\mathrm{e})}\right| \\
& \sim \frac{1}{M_{\mathrm{e}}\left(1+\left(\rho_{\mathrm{n}} / \rho\right)^{4} M_{\mathrm{i}, \text { eff }}^{2}\right)}
\end{aligned}
$$

$M_{\mathrm{e}}$ and $M_{\mathrm{i}, \text { eff }}$ increase monotonically with height, reaching unity at $z \sim 300 \mathrm{~km}$ and $z \sim 800 \mathrm{~km}, \rho_{\mathrm{n}} / \rho \sim 1$ in the photosphere and chromosphere, and $\left(\rho_{\mathrm{n}} / \rho\right)^{4} M_{\mathrm{i} \text {, eff }}^{2}$ rapidly decreases to $\ll 1$ in the transition region. Then Eq. (95) implies the following. For $z \lesssim 800 \mathrm{~km},\left|J_{\mathrm{P}}^{(\mathrm{e})} / J_{\mathrm{H}}^{(\mathrm{e})}\right| \sim M_{\mathrm{e}}^{-1}$. For $z$ between $\sim 800 \mathrm{~km}$ and the transition region, $\left|J_{\mathrm{P}}^{(\mathrm{e})} / J_{\mathrm{H}}^{(\mathrm{e})}\right| \sim 1 /\left(M_{\mathrm{e}}\left(1+M_{\mathrm{i} \text {, eff }}^{2}\right)\right)$. Above the transition region $\left|J_{\mathrm{P}}^{(\mathrm{e})} / J_{\mathrm{H}}^{(\mathrm{e})}\right| \sim M_{\mathrm{e}}^{-1}$. The behavior of the ratio for the ions is more complicated as indicated by Eqs. (59) and (60). However, some general properties of this behavior are determined analytically as follows. $M_{\mathrm{i} \text {, eff }} \gtrsim 1$ for $z \gtrsim 800 \mathrm{~km}$. Then between $z \sim 800 \mathrm{~km}$ and the transition region $\left|J_{\mathrm{P}}^{(\mathrm{i}, \text { eff })} / J_{\mathrm{H}}^{(\mathrm{i}, \text { eff })}\right|=\left|\sigma_{\mathrm{P}}^{(\mathrm{i}, \text { eff })} / \sigma_{\mathrm{H}}^{(\mathrm{i}, \text { eff })}\right| \sim M_{\mathrm{i}, \text { eff }}^{-1} \lesssim 1$, and decreases rapidly with height. Above the transition region $\Gamma \ll 1$ and $\rho_{\mathrm{n}} / \rho \ll 1$, in which case $\left|J_{\mathrm{P}}^{(\mathrm{i}, \text { eff })} / J_{\mathrm{H}}^{(\mathrm{i}, \text { eff })}\right| \sim$ $\left(m_{\mathrm{p}} / 2 m_{\mathrm{e}}\right)^{1 / 2}\left(\rho_{\mathrm{n}} / \rho\right) M_{\mathrm{p}}$, which is found to be $\sim 1$.
Figure 10 shows how $Q$ varies with $z$ for a range of values of $B(0)$. For $B(0) \lesssim 1500 \mathrm{G}$, and as discussed for the case of $B(0)=500 \mathrm{G}$ in the context of Fig. 4, Pedersen current dissipation is not significantly different from parallel current dissipation in the region below $H_{T \text { min }}$ where $Q$ is large since there $\sigma_{\mathrm{P}} / \sigma_{\|} \sim 1$. Even for $B(0)=3000 \mathrm{G}$, which is comparable to sunspot field strengths, the magnetizations are sufficiently small at $z=0$ to make $Q(0)$ moderately large. Then Pedersen current dissipation as distinct from parallel current dissipation in the sense that $\sigma_{\mathrm{P}} \ll \sigma_{\|}$is not important in the photosphere.

Figure 10 also shows that for $100 \leq B(0) \leq 3000 \mathrm{G}$ the heights of the corresponding local minima $Q_{\min }$ in $Q(z)$ are in the height range $\Delta H \sim 300-800 \mathrm{~km}$, centered near $H_{T \text { min }}$. For this range of $B(0)$ it is found that the height at which $Q=Q_{\text {min }}$ is essentially the height at which $M_{\mathrm{e}} M_{\mathrm{i} \text {, eff }}=1$. A value of

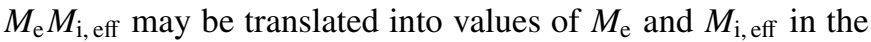
following way. As discussed in the context of Fig. 6, in the photosphere and chromosphere the collisional momentum transfer to the effective ions is essentially due to effective ion-neutral particle collisions. It follows that $M_{\mathrm{i}, \text { eff }} \sim\left(m_{\mathrm{e}} / m_{(\mathrm{i}, \text { eff }) \mathrm{n}}\right)^{1 / 2} M_{\mathrm{e}}$. The effective ions are a mixture of proxy ions and protons. In $\Delta H, m_{\mathrm{p}} \leq m_{\mathrm{i}, \text { eff }} \leq 37.3 m_{\mathrm{p}}$. Since the neutral species mass in $\Delta H$ is $\sim 1.27 m_{\mathrm{p}}$, it follows that $0.02 \lesssim\left(m_{\mathrm{e}} / m_{(\mathrm{i}, \text { eff }) \mathrm{n}}\right)^{1 / 2} \lesssim 0.03$. Then it is a good approximation to take $M_{\mathrm{i}, \text { eff }} \sim 0.025 M_{\mathrm{e}}$ in $\Delta H$. Then $M_{\mathrm{e}} M_{\mathrm{i} \text {, eff }} \sim 40 M_{\mathrm{i} \text {, eff }}^{2}$, so that if $M_{\mathrm{e}} M_{\mathrm{i} \text {, eff }}=1$ then $M_{\mathrm{i}, \text { eff }} \sim 0.16, M_{\mathrm{e}} \sim 6.3$, and Eq. (89) implies that $\sigma_{\|} \sim 22 \sigma_{\mathrm{P}}$. 


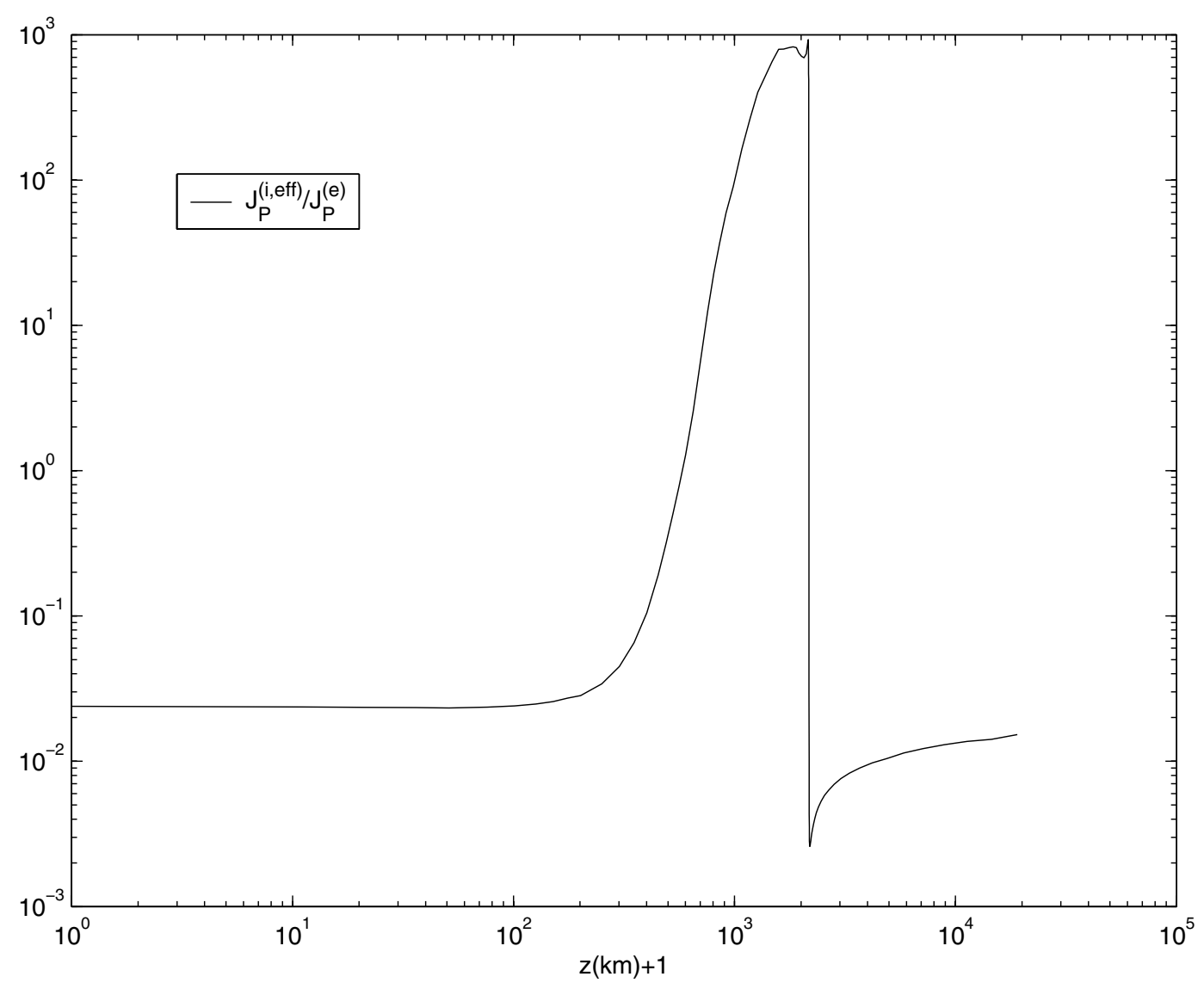

Fig. 5. Ratio of the effective ion Pedersen current density to the electron Pedersen current density as a function of height.

Since $M_{\mathrm{e}}$ and $M_{\mathrm{i} \text {, eff }}$ vary rapidly with height near the height of $Q_{\min }$, and since the condition that Pedersen current dissipation be essentially different from parallel current dissipation is $\sigma_{\|} \gg \sigma_{\mathrm{P}}$, it follows from Eq. (89) that for $100 \leq B(0) \leq$ $3000 \mathrm{G}, \Delta H$ separates underlying plasma in which Pedersen current dissipation is not important from overlying plasma in which Pedersen current dissipation is an essentially different heating mechanism from parallel current dissipation. Figure 11 shows how $\sigma_{\|} / \sigma_{\mathrm{P}}$ varies with $z$ for the same values of $B(0)$ used for Fig. 10. Figure 10 shows that $Q$ rapidly increases to unity above the height of $Q_{\min }$. It follows that $\Delta H$ is a transition layer between underying plasma in which Pedersen current dissipation is not important from overlying plasma in which it is distinctly different from parallel current dissipation, and is efficient in the region where $\rho_{\mathrm{n}} \sim \rho$, which is the chromosphere. If $100 \leq B(0) \leq 1500 \mathrm{G}$ then $\Delta H \sim 400-800 \mathrm{~km}$.

When $\sigma_{\|} \gg \sigma_{\mathrm{P}}$ the question arises as to the conditions under which $q_{\mathrm{P}} \gg q_{\|} \cdot q_{\mathrm{P}} \gg q_{\|}$is equivalent to $\left|\boldsymbol{E}_{\perp}^{\prime}\right|^{2} /\left|\boldsymbol{E}_{\|}\right|^{2} \gg$ $\sigma_{\|} / \sigma_{\mathrm{P}}$, so that if $\sigma_{\|} / \sigma_{\mathrm{P}} \gg 1$ it is necessary that $\left|\boldsymbol{E}_{\perp}^{\prime}\right|^{2} /\left|\boldsymbol{E}_{\|}\right|^{2} \gg$ 1. Heuristically, this is expected to be the case since if $\sigma_{\|} \gg \sigma_{\mathrm{P}}$ it is expected that $\boldsymbol{E}_{\|}$is shorted out relative to $\boldsymbol{E}_{\perp}^{\prime}$, allowing $\left|\boldsymbol{E}_{\perp}^{\prime}\right|$ to build up to relatively large values. An estimate of the size of $\left|\boldsymbol{E}_{\perp}^{\prime}\right| /\left|\boldsymbol{E}_{\|}\right|$necessary to make $q_{\mathrm{P}} \gg q_{\|}$in the chromosphere where $Q \sim 1$ is obtained from Fig. 3 as follows. At $z=10^{3} \mathrm{~km}$, $\sigma_{\|} \sim 10^{2} \sigma_{\mathrm{P}}$. Then it is necessary that $\left|\boldsymbol{E}_{\perp}^{\prime}\right| /\left|\boldsymbol{E}_{\|}\right| \gg 10$ in order that $q_{\mathrm{P}} \gg q_{\|}$.
The strong correlation between the observed height dependence of the chromospheric temperature rise and the predicted behavior of the particle magnetizations and their dependent quantities $Q$ and $\sigma_{\mathrm{P}} / \sigma_{\|}$support the hypothesis that Pedersen current dissipation in magnetic structures with $B(0) \gtrsim 100 \mathrm{G}$ causes the chromospheric temperature inversion.

\section{Independent calculation of $Q$ from the photosphere through the lower chromosphere}

Figure 6 shows that the plasma is weakly ionized from the photosphere up to $z \sim 1000 \mathrm{~km}$ in the sense that the effective collision frequencies of electrons, protons, and the proxy metallic ion with the neutral component of the plasma are much greater than with the unlike charged species components of the plasma. Then over this height range, where most chromospheric heating occurs, $Q$ is accurately given by its value for a weakly ionized plasma. It is proved in this section that for an $N$ species, weakly ionized plasma, $Q$ rapidly approaches unity as the charged particles become magnetized. $N$ is arbitrary. Then regardless of the composition of the plasma, $Q \rightarrow 1$ as the charged particles become magnetized. The quasi-neutrality condition plays a critical role in the proof. The proof is as follows. 


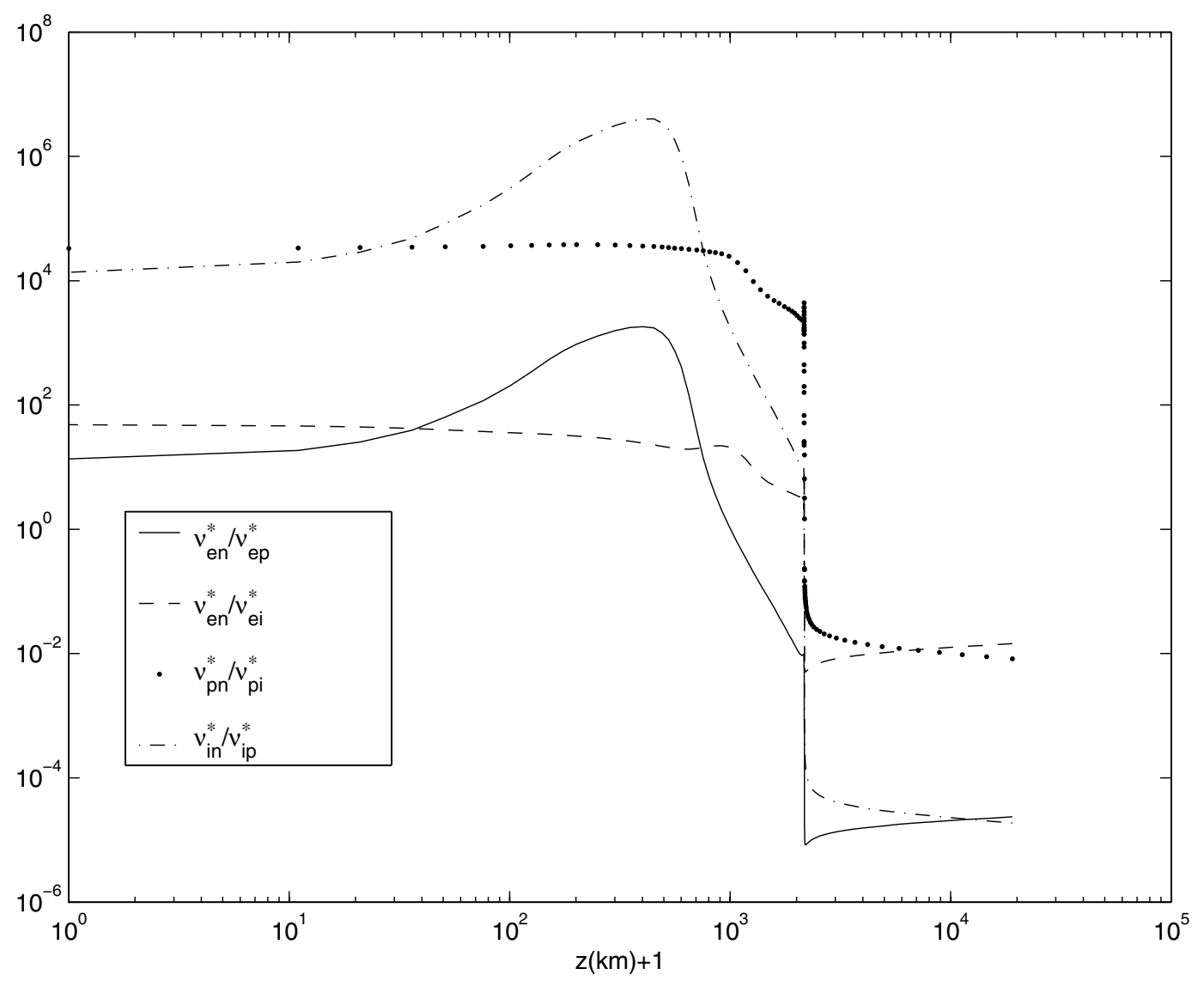

Fig. 6. Ratios of charged-neutral to charged-charged reduced collision frequencies for electrons, protons, and proxy ions as functions of height.

For an $N$ species weakly ionized plasma the electrical conductivities are given by (Mitchner \& Kruger 1973)

$$
\begin{gathered}
\sigma_{\mathrm{P}}=\sum_{s=1}^{N} \sigma_{\mathrm{P}}^{(s)} \\
\sigma_{\mathrm{H}}=\sum_{s=1}^{N} \sigma_{\mathrm{H}}^{(s)}
\end{gathered}
$$

where

$\sigma_{\mathrm{P}}^{(s)}=\frac{\sigma_{\|}^{(s)}}{1+M_{s}^{2}}$

$\sigma_{\mathrm{H}}^{(s)}=\frac{\operatorname{sign}\left(q_{s}\right) \sigma_{\|}^{(s)} M_{s}}{1+M_{s}^{2}}$

$\sigma_{\|}^{(s)}=\frac{n_{s} q_{s}^{2}}{m_{s} v_{s}^{*}}$

For $M_{s}^{2} \gg 1$,

$$
\sigma_{\mathrm{P}} \rightarrow \sum_{s=1}^{N} \frac{\sigma_{\|}^{(s)}}{M_{s}^{2}}
$$

$$
\begin{aligned}
\sigma_{\mathrm{H}} & \rightarrow \sum_{s=1}^{N} \operatorname{sign}\left(q_{s}\right) \sigma_{\|}^{(s)}\left(\frac{1}{M_{s}}-\frac{1}{M_{s}^{3}}\right) \\
& =\frac{c}{B} \sum_{s=1}^{N} n_{s} q_{s}-\sum_{s=1}^{N} \frac{\operatorname{sign}\left(q_{s}\right) \sigma_{\|}^{(s)}}{M_{s}^{3}} \\
& =-\sum_{s=1}^{N} \frac{\operatorname{sign}\left(q_{s}\right) \sigma_{\|}^{(s)}}{M_{s}^{3}}
\end{aligned}
$$

where the first term in Eq. (103) vanishes by the quasineutrality condition

$\sum_{s=1}^{N} n_{s} q_{s}=0$

The condition that $M_{s}^{2} \gg 1$ is satisfied for $M_{s} \gtrsim 3$.

Then for $M_{s}^{2} \gg 1$, Eqs. (101) and (104), and Eq. (13) for $Q$ give

$$
\begin{aligned}
& Q \sim \frac{1}{1+\epsilon^{2}} \\
& \epsilon=\frac{\sum_{s=1}^{N} \frac{\operatorname{sign}\left(q_{s}\right) \sigma_{\|}^{(s)}}{M_{s}^{3}}}{\sum_{s=1}^{N} \frac{\sigma_{\|}^{(s)}}{M_{s}^{2}}} .
\end{aligned}
$$




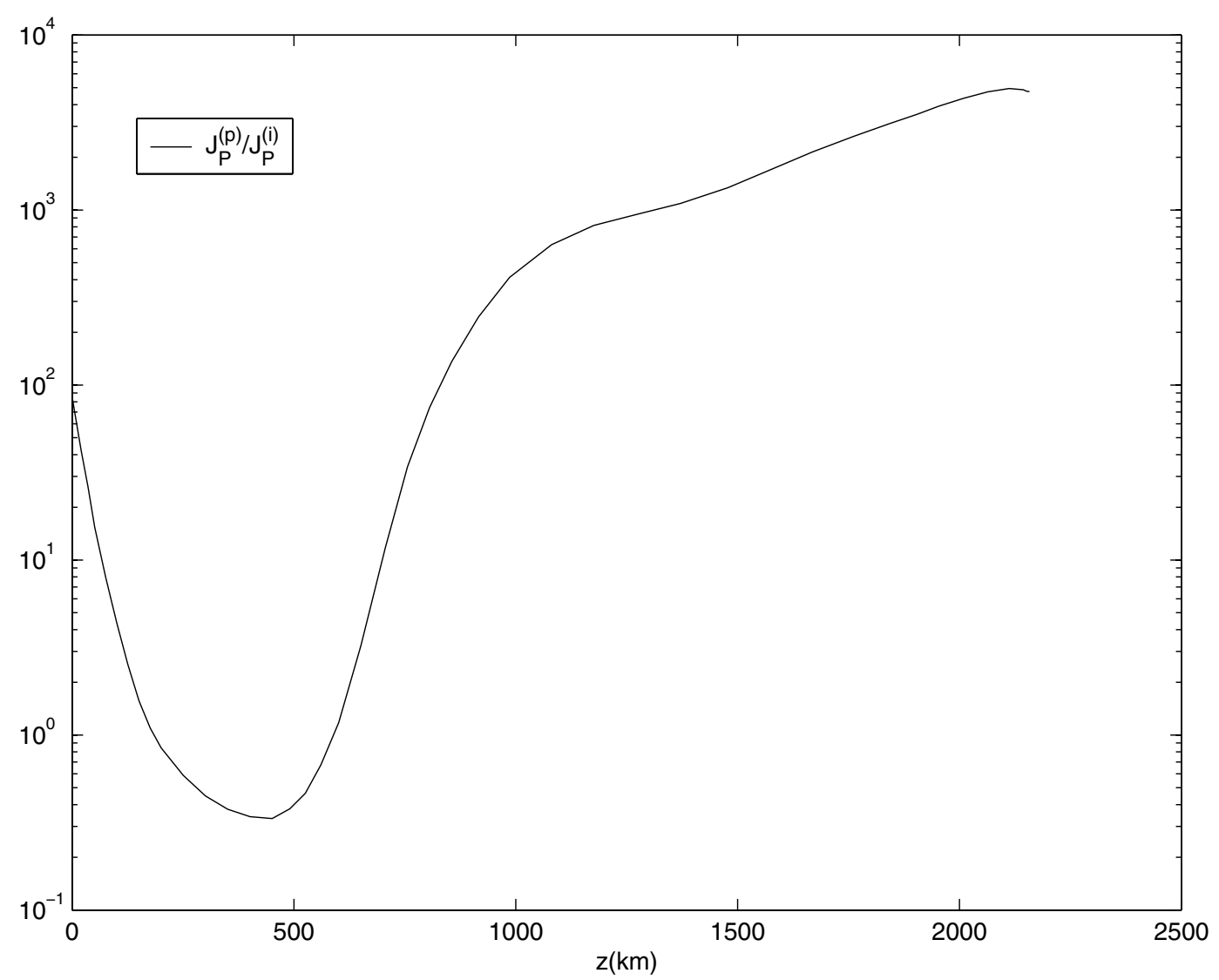

Fig. 7. Ratio of the proton Pedersen current density to the proxy ion Pedersen current density over the height range from 0 to $2157 \mathrm{~km}$.

Using the definitions of $\sigma_{\|}^{(s)}, M_{s}$, and $\omega_{s}$,

$\frac{\sigma_{\|}^{(s)}}{M_{s}^{2}}=\frac{c}{B}\left(\frac{n_{s}\left|q_{s}\right|}{M_{s}}\right) \equiv \frac{c}{B} A_{s}$.

Then

$$
\begin{aligned}
|\epsilon| & <\frac{\sum_{s=1}^{N} \frac{A_{s}}{M_{s}}}{\sum_{s=1}^{N} A_{s}}<\sum_{s=1}^{N} \frac{A_{s}}{M_{s} A_{s}}\left(\text { since } A_{s}>0\right) \\
& =\sum_{s=1}^{N} \frac{1}{M_{s}} .
\end{aligned}
$$

Therefore, for $M_{s} \rightarrow \infty$ one has $|\epsilon| \rightarrow 0$ and

$$
1 \geq Q \rightarrow 1-\epsilon^{2}>1-\left(\sum_{s=1}^{N} \frac{1}{M_{s}}\right)^{2} \rightarrow 1,
$$

which implies that $Q \rightarrow 1$ as was to be proved.

This result implies that magnetizing a weakly ionized plasma allows it to be efficiently heated by any mechanism that drives Pedersen current. This result is consistent with the behavior of $Q$ from the photosphere to the upper chromosphere shown in Figs. 4 and 10, and based on the conductivity model in Sect. 2.2. This is expected since Fig. 5 shows that the effective ions carry the Pedersen current, and Fig. 6 shows that the protons and proxy ions, of which the effective ion is comprised, scatter as in a weakly ionized plasma.

If the quasi-neutrality condition is not enforced then the first term in Eq. (103) does not vanish, and $Q \rightarrow 0$ as $M_{s} \rightarrow \infty$.

\section{Comments on the connection between $q_{\mathrm{P}} / q_{\|}$ and the degree to which $B$ is force-free}

By definition, a magnetic field is force-free if $\boldsymbol{J} \times \boldsymbol{B}=0$, in which case $\boldsymbol{J}$ is parallel to $\boldsymbol{B}$, and $q_{\mathrm{P}}=0$. Force-free magnetic fields are sometimes used to model the magnetic field in the solar atmosphere. Observations and semi-empirical modeling suggest that the magnetic field in the solar atmosphere is not force-free near the photosphere but becomes approximately force-free $\sim 300-400 \mathrm{~km}$ above the photosphere, and remains so in the overlying atmosphere (Gary 1989; Metcalf et al. 1995; Pevtsov et al. 1997; Moon et al. 2002).

The degree to which a magnetic field is force-free is defined by

$$
\begin{aligned}
D_{\mathrm{ff}} & \equiv \frac{J_{\|}}{J} \\
& =\frac{1}{\left(1+\frac{J_{\mathrm{P}}^{2}+J_{\mathrm{H}}^{2}}{J_{\|}^{2}}\right)^{1 / 2}} \\
& =\frac{1}{\left(1+\frac{\left(\sigma_{\mathrm{P}}^{2}+\sigma_{\mathrm{H}}^{2}\right) E_{\perp}^{\prime}}{\sigma_{\|}^{2} E_{\|}^{2}}\right)^{1 / 2}} .
\end{aligned}
$$

$0 \leq D_{\mathrm{ff}} \leq 1$ and $\cos ^{-1}\left(D_{\mathrm{ff}}\right)$ is the angle $\boldsymbol{J}$ makes with $\boldsymbol{B}$.

An equation is now derived for $q_{\mathrm{P}} / q_{\|}$as a function of $D_{\mathrm{ff}}, M_{\mathrm{e}} M_{\mathrm{i}, \text { eff }}$, and $\rho_{\mathrm{n}} / \rho$. 


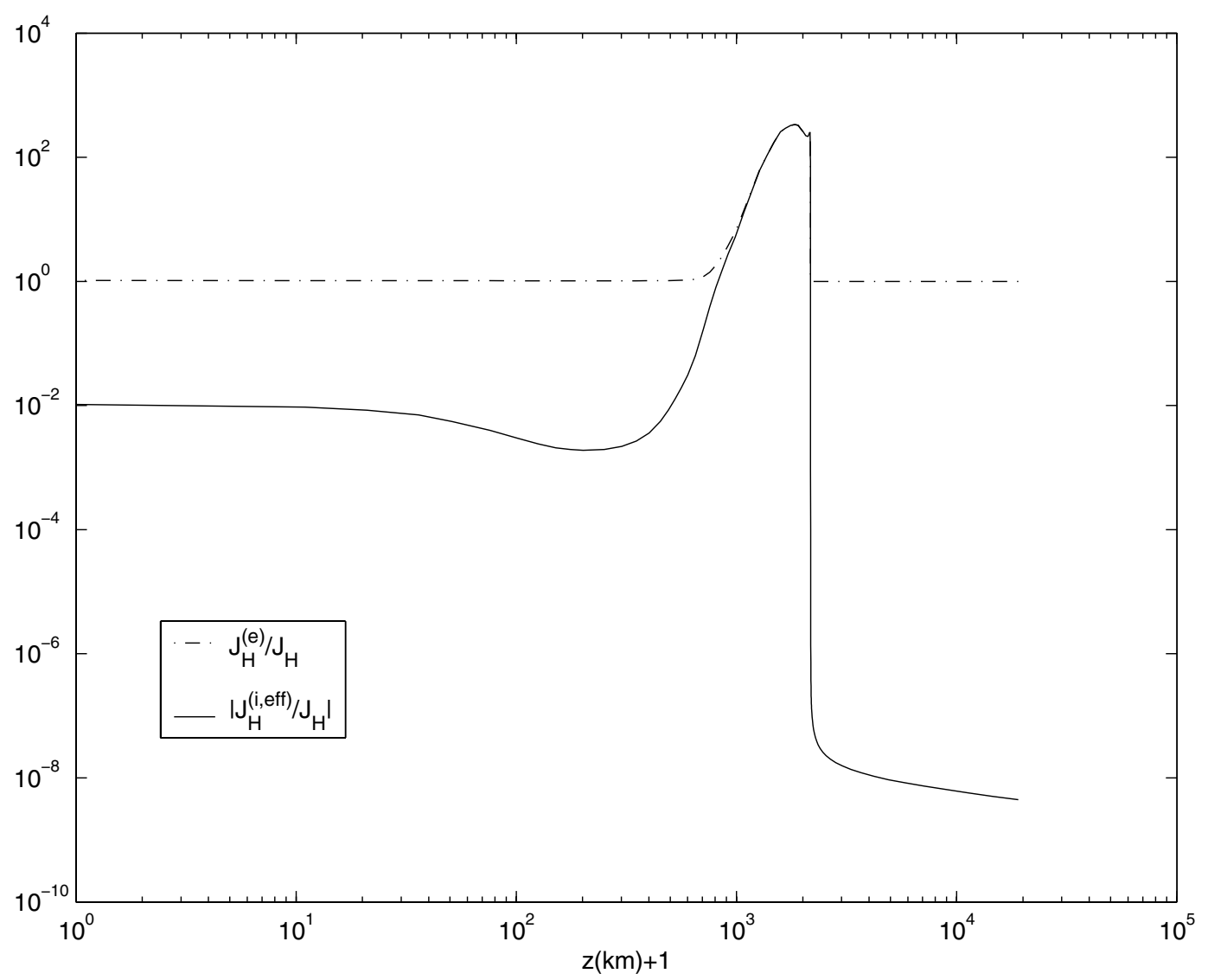

Fig. 8. Hall current density for electrons and effective ions, normalized to the total Hall current density, as a function of height.

One has

$\frac{q_{\mathrm{P}}}{q_{\|}}=\frac{\sigma_{\mathrm{P}}}{\sigma_{\|}}\left(\frac{E_{\perp}^{\prime}}{E_{\|}}\right)^{2}$.

Solving Eq. (114) for $\left(E_{\perp}^{\prime} / E_{\|}\right)^{2}$, Eq. (115) may be written as

$\frac{q_{\mathrm{P}}}{q_{\|}}=\left(\frac{1}{D_{\mathrm{ff}}^{2}}-1\right) \frac{\sigma_{\mathrm{P}} \sigma_{\|}}{\sigma_{\mathrm{P}}^{2}+\sigma_{\mathrm{H}}^{2}}$.

Using the form of the conductivities in Sect. 2.2.4, Eq. (116) becomes

$\frac{q_{\mathrm{P}}}{q_{\|}}=\left(\frac{1}{D_{\mathrm{ff}}^{2}}-1\right)\left(1+\left(\frac{\rho_{\mathrm{n}}}{\rho}\right)^{2} M_{\mathrm{e}} M_{\mathrm{i}, \mathrm{eff}}\right)$.

It follows from Eq. (117) that since $\rho_{\mathrm{n}} / \rho \sim 1$ in the chromosphere, if $M_{\mathrm{e}} M_{\mathrm{i}, \text { eff }} \gg 1$ then $q_{\mathrm{P}} \gg q_{\|}$in the chromosphere unless $D_{\mathrm{ff}}$ is essentially exactly unity. It also follows from this equation that if a magnetic field configuration is non-force-free in that $D_{\text {ff }} \ll 1$ then $q_{\mathrm{P}} \gg q_{\|}$.

For $B(0)=1500 \mathrm{G}, M_{\mathrm{e}}(0) M_{\mathrm{i}, \text { eff }}(0)=0.0156$. Values of $M_{\mathrm{e}}(0) M_{\mathrm{i}, \text { eff }}(0)$ for other field strengths are obtained by noting that $M_{\mathrm{e}} M_{\mathrm{i} \text {, eff }} \propto B^{2}$. For $B(0)=(100,200,500,1000,1500) \mathrm{G}$, $M_{\mathrm{e}} M_{\mathrm{i} \text {, eff }}$ increases slowly with height below $H_{T \text { min }}$, and reaches unity at $z=(817,710,583,479,421) \mathrm{km}$. Then, even for the highest photospheric magnetic field strengths, $M_{\mathrm{e}} M_{\mathrm{i} \text {, eff }} \ll 1$ for almost all of the plasma below $H_{T \text { min }}$. For $M_{\mathrm{e}} M_{\mathrm{i} \text {, eff }} \ll 1$, $D_{\mathrm{ff}} \sim\left(1+q_{\mathrm{P}} / q_{\|}\right)^{-1 / 2}$. Then a measurement of $D_{\mathrm{ff}}$ determines $q_{\mathrm{P}} / q_{\|}$. If, in addition to $M_{\mathrm{e}} M_{\mathrm{i}, \text { eff }} \ll 1$, it is the case that $M_{\mathrm{e}} \ll 1$ then this measurement also determines $E_{\perp}^{\prime} / E_{\|}$since $q_{\mathrm{P}} / q_{\|}=$ $\sigma_{\mathrm{P}} E_{\perp}^{\prime} / \sigma_{\|} E_{\|}$, and $\sigma_{\mathrm{P}} \sim \sigma_{\|}$according to Eq. (89).

If $M_{\mathrm{e}} M_{\mathrm{i}, \text { eff }} \gg 1$ then Eq. (117) implies that $q_{\mathrm{P}} / q_{\|}<1$ only when $D_{\mathrm{ff}}>1-1 /\left(2 M_{\mathrm{e}} M_{\mathrm{i} \text {, eff }}\right)+$ terms of order $\left(1 / M_{\mathrm{e}} M_{\mathrm{i}, \text { eff }}\right)^{2}$. For example, if $M_{\mathrm{e}} M_{\mathrm{i}, \text { eff }}=5$ then $q_{\mathrm{P}} / q_{\|}<1$ only if $D_{\mathrm{ff}}>0.9$, and if $M_{\mathrm{e}} M_{\mathrm{i} \text {, eff }}=50$ then $q_{\mathrm{P}} / q_{\|}<1$ only if $D_{\mathrm{ff}}>0.99$. The rapid increase of $M_{\mathrm{e}} M_{\mathrm{i} \text {, eff }}$ with height to values orders of magnitude larger than unity in the lower chromosphere implies that $q_{\mathrm{P}}$ is orders of magnitude greater than $q_{\|}$in the chromosphere unless $\boldsymbol{B}$ is essentially exactly force free. For $B(0)=(100,200,500,1000,1500) \mathrm{G}, M_{\mathrm{e}} M_{\mathrm{i}, \text { eff }}=50$ for $z=$ $(1871,1211,896,757,701) \mathrm{km}$, and $M_{\mathrm{e}} M_{\mathrm{i}, \text { eff }}$ increases rapidly by orders of magnitude above these heights.

The magnetic field in any given region of the solar atmosphere is not exactly force-free, so it is always the case that $q_{\mathrm{P}}>0$. The question is whether this heating rate is a significant fraction of the net radiative loss. This question can only be answered by knowing $\boldsymbol{E}_{\perp}^{\prime}$. The MHD wave heating model in Goodman (2000) computes $\boldsymbol{E}_{\perp}^{\prime}$, and predicts that at a height near the middle of the lower chromospheric network $J_{\|} / J \ll 1$ within a cylindrical region with a horizontal diameter $\sim 10 \mathrm{~km}$. Then the magnetic field in this region is highly non-force-free. The model also predicts that $q_{\mathrm{P}} \sim 10^{6.5}-10^{9.5} q_{\|}$, and balances the net radiative loss from the chromosphere in this region. These predictions are consistent with Eq. (117), and suggest that on sub-resolution spatial scales Pedersen current 


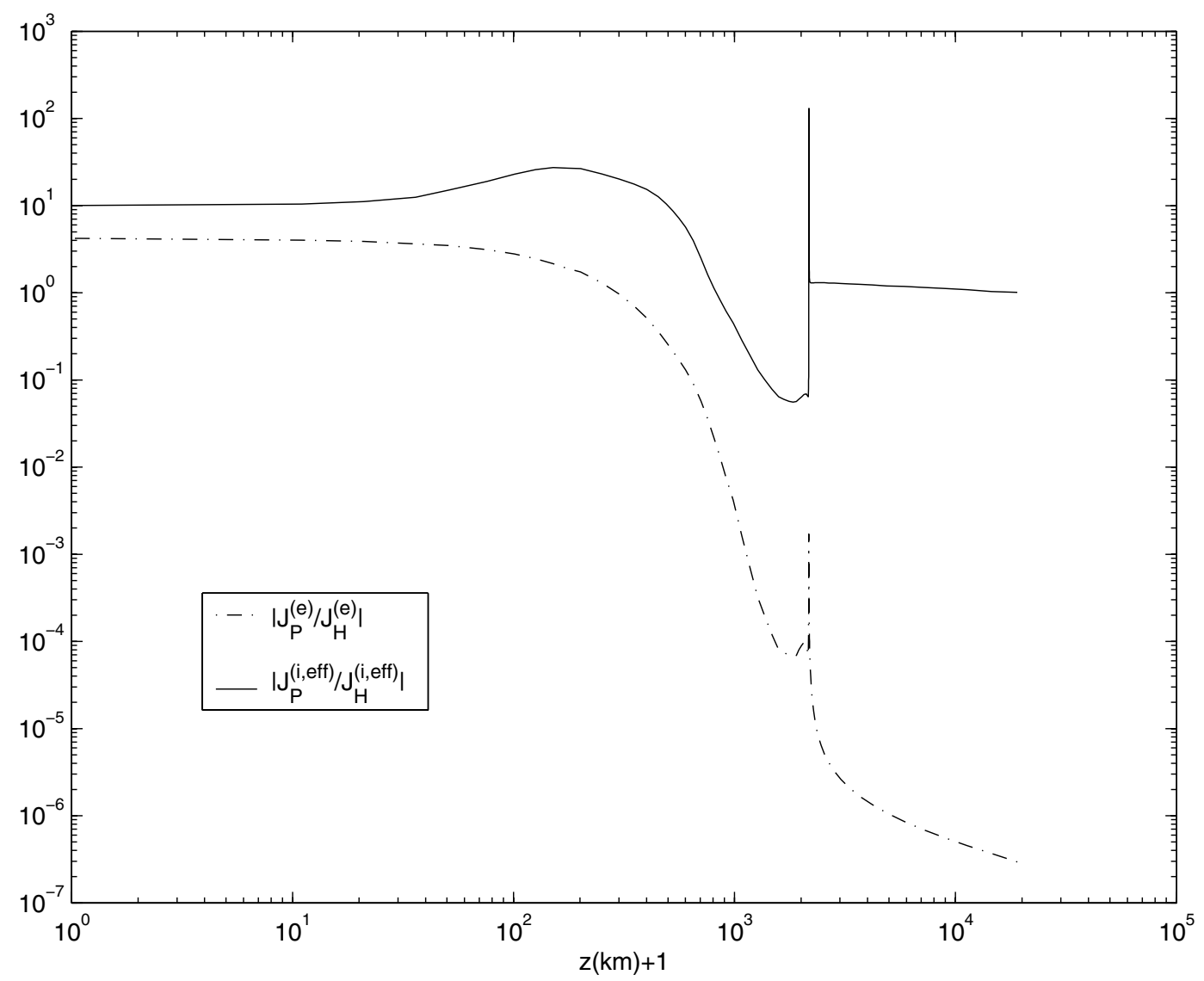

Fig. 9. Ratio of Pedersen current density to Hall current density for electrons and effective ions as a function of height.

dissipation in the presence of non-force-free magnetic fields makes a major contribution to chromospheric heating.

\section{Conclusions}

Pedersen current dissipation is an efficient mechanism for dissipating the energy in electric current driven orthogonal to the magnetic field in non-flaring regions of the solar chromosphere. Any process that drives current orthogonal to the magnetic field is damped by this mechanism to the degree to which its energy is channeled into generating and maintaining the CM electric field that drives this current. Pedersen current dissipation is a highly inefficient mechanism for heating the transition region and corona on the spatial scales of the FAL models. Pedersen current dissipation is not important in the photosphere since although $Q \sim 1$ in the photosphere for $B \lesssim 1500 \mathrm{G}$, the particle magnetizations are so small that the conductivity tensor is nearly isotropic, implying there is no essential distinction between Pedersen and magnetic field aligned current dissipation.

A necessary condition for Pedersen current dissipation to be important is that $\sigma_{\mathrm{P}} \ll \sigma_{\|}$, which means that it is an essentially different heating mechanism from parallel current dissipation. This condition is equivalent to $M_{\mathrm{e}} \gg 1$. Given that this condition is satisfied, Pedersen current dissipation is efficient if $Q \sim 1$, which is equivalent to the condition that $\sigma_{\mathrm{P}} \gg \sigma_{\mathrm{H}}$. This condition is satisfied when the effective ions are magnetized in the sense that $M_{\mathrm{i} \text {, eff }} \gtrsim 2$. For $100 \leq B(0) \leq 3000 \mathrm{G}$ there exists a transition layer in the height range $\Delta H \sim 300-800 \mathrm{~km}$ that separates the lower, weakly magnetized atmosphere where Pedersen current dissipation is not important from the upper, strongly magnetized atmosphere where $\sigma_{\mathrm{H}} \ll \sigma_{\mathrm{P}} \ll \sigma_{\|}$, and hence Pedersen current dissipation can be important, in the region where the plasma is mostly neutral $\mathrm{H}$, which is the chromosphere. If $100 \leq B(0) \leq 1500 \mathrm{G}$ then $\Delta H \sim 400-800 \mathrm{~km}$. The transition layer may be the origin of the single height temperature minimum that appears in horizontally space averaged models such as FAL CM. The averaging is applied to the observed emission, and so implicitly includes averaging over a group of magnetic field configurations with a range of photospheric field strengths.

For $z \gtrsim 600 \mathrm{~km}$ the protons carry all of the Pedersen current in the chromosphere. The rapid increase of $M_{\mathrm{p}}$ with height that occurs in the transition layer transforms $J_{\perp}$ from being mainly a Hall current, which is not dissipative, into being almost entirely a Pedersen current, maximizing the rate at which the energy in $J_{\perp}$ is thermalized. Equivalently, the direction of $\boldsymbol{J}_{\perp}$ is rapidly transformed from being orthogonal to $\boldsymbol{E}_{\perp}^{\prime}$ to being parallel to $\boldsymbol{E}_{\perp}^{\prime}$. These conclusions are independent of the geometry of the magnetic field since the magnetization depends on the magnetic field only through its magnitude.

In this paper the heating rate due to Pedersen current dissipation driven by a specific process such as MHD waves or quasi-steady flows is not computed. Only the heating efficiency $Q$ is computed. A model that computes $\boldsymbol{E}^{\prime}$ is 


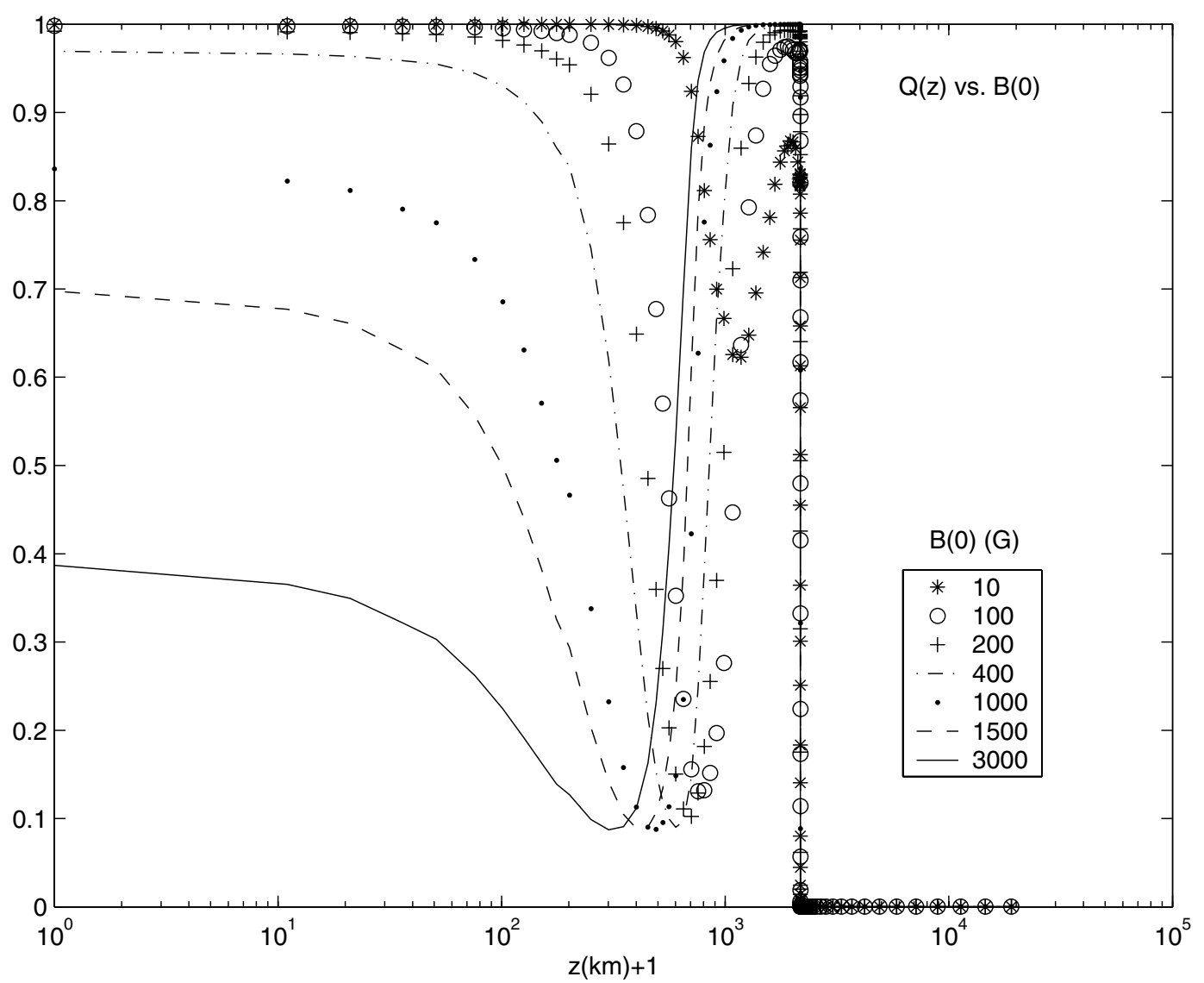

Fig. 10. Variation of heating efficiency with height for several photospheric magnetic field strengths.

necessary in order to compute $q_{\mathrm{P}}$ and $q_{\|}$. However, using the model in this paper an estimate of the size of $E_{\perp}^{\prime}$ necessary to drive Pedersen current dissipation that balances the net radiative loss from the chromosphere is obtained as follows. Anderson \& Athay (1989a,b) estimate the heating rate per unit mass, $q_{m}$, in the height range of $1000-2000 \mathrm{~km}$ to be constant and equal to $4.5 \times 10^{9} \mathrm{ergs}^{-1} \mathrm{~s}^{-1}$. If this heating rate is due entirely to Pedersen current dissipation then $E_{\perp}^{\prime}=\left(\rho q_{m} / \sigma_{\mathrm{P}}\right)^{1 / 2}$. Evaluating this expression for $B(0)=500 \mathrm{G}$ it is found that $E_{\perp}^{\prime}$ decreases monotonically from $\sim 0.269 \mathrm{~V} \mathrm{~m}^{-1}$ at $z=1000 \mathrm{~km}$ to $\sim 0.012 \mathrm{~V} \mathrm{~m}^{-1}$ at $z=2000 \mathrm{~km}$. If, in addition, $E_{\perp}^{\prime}$ is a convection electric field then $U_{\perp}=c E_{\perp}^{\prime} / B$ is the $\mathrm{CM}$ flow speed perpendicular to the magnetic field that generates this electric field. Again for $B(0)=500 \mathrm{G}$ it is found that $U_{\perp} \mathrm{de}$ creases monotonically from $\sim 43.9 \mathrm{~m} \mathrm{~s}^{-1}$ at $z=1000 \mathrm{~km}$ to $\sim 13.5 \mathrm{~m} \mathrm{~s}^{-1}$ at $z=2000 \mathrm{~km}$. These values of $E_{\perp}^{\prime}$ and $U_{\perp}$ are consistent with those predicted by Goodman $(2000,2001)$ for MHD wave driven heating by Pedersen current dissipation in the lower chromosphere.

If, as proposed here, Pedersen current dissipation is a major source of chromospheric heating then the preceding conclusions are extended as follows. The lower boundary of the chromosphere varies over the surface of the Sun. This boundary is lower in regions with higher magnetic field strength. Then the chromosphere begins lower in the atmosphere in active and network regions than in internetwork regions. The fact that the filling factor of kilogauss strength magnetic field concentrations in network and active regions is larger than in internetwork regions accounts for the fact that the net radiative loss from network and active regions is several times larger than from internetwork regions, although these losses are of the same order of magnitude.

These conclusions are consistent with the MHD wave heating mechanism proposed by Goodman (2000, 2001). For that mechanism the Pedersen currents are driven by upward propagating slow magnetoacoustic waves generated in magnetic flux tubes with photospheric field strengths $\sim 10^{2}-10^{3} \mathrm{G}$. The center of mass flow driven by the waves has a component perpendicular to $\boldsymbol{B}$. This component generates a convection electric field $(\boldsymbol{U} \times \boldsymbol{B}) / \boldsymbol{c}$ that adds to the wave induction electric field $\boldsymbol{E}$ to create the CM electric field. The convection electric field dominates the induction electric field in driving the Pedersen current. The mechanical energy that is thermalized to balance the net radiative loss from the chromosphere is provided by wave driven convection. The waves do not experience significant dissipation until they reach the height in a flux tube at which the magnetization of the protons begins to rapidly increase with height, causing the rapid transformation of $\boldsymbol{J}_{\perp}$ from a Hall current into a Pedersen current. Goodman (2000) estimates that near the middle of the lower chromosphere the horizontal extent of the region in a flux tube in which $\sim 90 \%$ of the heating occurs is $\sim 10 \mathrm{~km}$. This is consistent with the MISMA (Micro-Structured Magnetic Atmosphere) semi-empirical model estimates that 


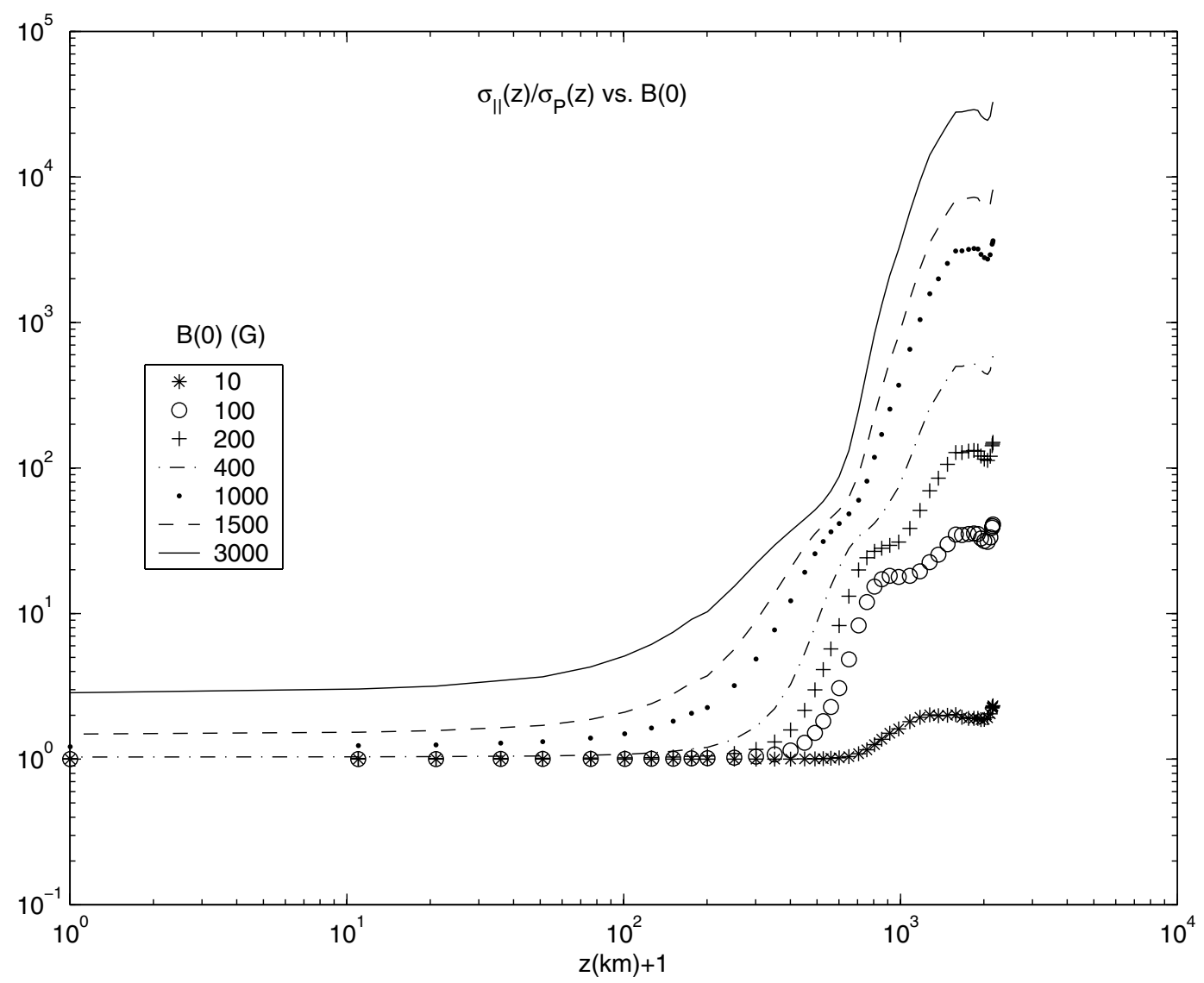

Fig. 11. Variation of the ratio of the parallel conductivity to the Pedersen conductivity as a function of height for the same photospheric magnetic field strengths used for Fig. 10.

most of the unsigned magnetic flux through the photosphere is in the form of magnetic flux tubes with sub-resolution diameters as small as a few kilometers, and field strengths $\sim 10^{2}-10^{3}$ G (Sánchez Almeida \& Landi degl'Innocenti 1996; Sánchez Almeida et al. 1996; Sánchez Almeida 1997, 2000; Sánchez Almeida \& Lites 2000; Sánchez Almeida et al. 2001; Socas-Navarro \& Sánchez Almeida 2002).

If a magnetic field is largely non-force-free in that $J_{\|} / J \ll 1$ then $q_{\mathrm{P}} \gg q_{\|}$. Due to the large value of $M_{\mathrm{e}} M_{\mathrm{i}, \text { eff }}$ in the chromosphere, $q_{\mathrm{P}} \gg q_{\|}$throughout the chromosphere except for magnetic fields that are virtually exactly force-free. If a magnetic field is exactly force-free then $q_{\mathrm{P}}=0$. If it is highly but not exactly force-free then $q_{\mathrm{P}}$ might still be significant.

The electrical conductivity of the solar atmosphere is not a constant or a scalar. It is a highly anisotropic tensor with components that can differ and vary by orders of magnitude with position and time. This must be taken into account in any realistic model of a resistive heating mechanism.

Pedersen current dissipation is a fundamental dissipative process in plasmas since the heating rate $q_{\mathrm{P}}=\boldsymbol{J} \cdot\left(\boldsymbol{E}_{\perp}+(\boldsymbol{U} \times\right.$ $\boldsymbol{B}) / c$ ) irrespective of the processes that determine $\boldsymbol{J}, \boldsymbol{E}_{\perp}, \boldsymbol{B}$, and $\boldsymbol{U}$.

Acknowledgements. This work was supported by NSF grant ATM 9816335 to the Catholic University of America, NSF grant ATM 0242820 to the Institute for Scientific Research, and by a HUDEDI grant to the Institute for Scientific Research. The author thanks
Eugene Avrett and Rudolf Loeser of the Harvard-Smithsonian Center for Astrophysics for providing numerical solutions to the FAL models.

\section{References}

Anderson, L. S., \& Athay, R. G. 1989a, ApJ, 336, 1089 Anderson, L. S., \& Athay, R. G. 1989b, ApJ, 346, 1010 Aschwanden, M. J. 2001, ApJ, 560, 1035

Avrett, E. H., \& Loeser, R. 2001, private communication Balescu, R. 1988, Transport Processes in Plasmas, 1, North - Holland Berger, T. E., \& Title, A. M. 2001, ApJ, 553, 449

Bianda, M., Stenflo, J. O., \& Solanki, S. K. 1998, A\&A, 337, 565

Book, D. L. 1994, NRL Plasma Formulary (Naval Research Laboratory, Washington, DC)

Braginskii, S. I. 1965 Reviews of Plasma Physics, ed. M. A. Leontovich, 1, 205 (Consultants Bureau)

Carlsson, M., Judge, P. G., \& Wilhelm, K. 1997, ApJ, 486, L63

Carlsson, M., \& Stein, R. F. 1997, ApJ, 481, 500

Carlsson, M., \& Stein, R. F. 2002, ApJ, 572, 626

Cattaneo, F. 1999, ApJ, 515, L39

Cattaneo, F., Lenz, D., \& Weiss, N. 2001, ApJ, 563, L91

Chapman, S., \& Cowling, T. J. 1970, The Mathematical Theory of Non-Uniform Gases (Cambridge: University Press)

Curdt, W., \& Heinzel, P. 1998, ApJ, 503, L95

Domínguez Cerdeña, I., Kneer, F., \& Sánchez Almeida, J. 2003, ApJ, 582, L55

Durney, B. R., de Young, D. S., \& Roxburgh, I. W. 1993, Sol. Phys., 145,207

Emonet, T., \& Cattaneo, F. 2001, ApJ, 560, L197 
Faurobert, M., Arnaud, J., Vigneau, J., \& Frisch, H. 2001 A\&A, 378, 627

Faurobert-Scholl, M. 1993, A\&A, 268, 765

Faurobert-Scholl, M. 1994, A\&A, 285, 655

Faurobert-Scholl, M., Feautrier, N., Machefert, F., Petrovay, K., \& Spielfiedel, A. 1995, A\&A, 298, 289

Fontenla, J. M., Avrett, E. H., \& Loeser, R. 2002, ApJ, 572, 636

Gary, G. A. 1989, ApJS, 69, 323

Goodman, M. L. 1998, ApJ, 503, 938

Goodman, M. L. 2000, ApJ, 533, 501

Goodman, M. L. 2001, Space Sci. Rev., 95, 79

Grossmann-Doerth, U., Keller, C. U., \& Schussler, M. 1996, A\&A, 315,610

Hasan, S. S., \& Kalkofen, W. 1999, ApJ, 519, 899

Hasan, S. S., Kalkofen, W., \& van Ballegooijen, A. A. 2000, ApJ, 535, L67

Hoekzema, N. M., Rutten, R. J., \& Cook, J. W. 1997, ApJ, 474, 528

Ichimaru, S. 1973, Basic Principles of Plasma Physics, (Philippines: W. A. Benjamin)

Judge, P. G., \& Carpenter, K. G. 1998, ApJ, 494, 828

Judge, P. G., Tarbell, T. D., \& Wilhelm, K. 2001, ApJ, 554, 424

Kalkofen, W. 1997, ApJ, 486, L145

Kalkofen, W. 2001, ApJ, 557, 376

Keller, C. U., Deubner, F. -L., Egger, U., Fleck, B., \& Povel, H. P. 1994, A\&A, 286, 626

Krall, N. A., \& Trivelpiece, A.W. 1986, Principles of Plasma Physics, (San Francisco: San Francisco Press)

Krijger, J. M., Rutten, R. J., Lites, B. W., et al. 2001, A\&A, 379, 1052

Lin, H. 1995, ApJ, 446, 421

Lin, H., \& Rimmele, T. 1999, ApJ, 514, 448

Lites, B. W. 2002, ApJ, 573, 431

Lites, B. W., Rutten, R. J., \& Berger, T. E. 1999, ApJ, 517, 1013

McAteer, R. T. J., Gallagher, P. T., Williams, D. R., et al. 2002, ApJ, 567, L165

McIntosh, S. W., Bogdan, T. J., Cally, P. S., et al. 2001, ApJ, 548, $\mathrm{L} 237$

Metcalf, T. R., Jiao, L., McClymont, A. N., Canfield, R. C., \& Uitenbroek, H. 1995, ApJ, 439, 474
Mitchner, M., \& Kruger, C. H. 1973, Partially Ionized Gases (New York: Wiley)

Moon, Y. -J., Choe, G. S., Yun, H. S., Park, Y. D., \& Mickey, D. L. 2002, ApJ, 568, 422

Muller, R., Dollfus, A., Montagne, M., Moity, J., \& Vigneau, J. 2000, A\&A, 359, 373

Nindos, A., \& Zirin, H. 1998, Sol. Phys., 179, 253

Petrovay, K., \& Szakaly, G. 1993, A\&A, 274, 543

Pevtsov, A. A., Canfield, R. C., \& McClymont, A. N. 1997, ApJ, 481, 973

Sánchez Almeida, J. 1997, ApJ, 491, 993

Sánchez Almeida, J. 2000, ApJ, 544, 1135

Sánchez Almeida, J., \& Landi degl'Innocenti, E. 1996, Sol. Phys., 164,203

Sánchez Almeida, J., Landi degl'Innocenti, E., Martinez Pillet, V., \& Lites, B. W. 1996, ApJ, 466, 537

Sánchez Almeida, J., \& Lites, B. W. 2000, ApJ, 532, 1215

Sánchez Almeida, J., Asensio Ramos, A., Trujillo Bueno, J., \& Cernicharo, J. 2001, ApJ, 555, 978

Sigwarth, M. 2001, ApJ, 563, 1031

Sivaraman, K. R., Gupta, S. S., Livingston, W. C., et al. 2000, A\&A, 363,279

Sivaraman, K. R., \& Livingston, W. C. 1982, Sol. Phys., 80, 227

Socas-Navarro, H., \& Sánchez Almeida, J. 2002, ApJ, 565, 1323

Solanki, S. K., Zufferey, D, Lin, H., Rüedi, I., \& Kuhn, J. R. 1996, A\&A, 310, L33

Stenflo, J. O. 1982, Sol. Phys., 80, 209

Stenflo, J. O., Gandorfer, A., Holzreuter, R., et al. 2002, A\&A, 389, 314

Stenflo, J. O., Gandorfer, A., Wenzler, T., \& Keller C. U. 2001, A\&A, 367,1033

Stenflo, J. O., Keller, C. U., \& Gandorfer, A. 1998, A\&A, 329, 319

Vernazza, J. E., Avrett, E. H., \& Loeser, R. 1981, ApJS, 45, 635

Wikstol, O., Hansteen, V. H., Carlsson, M., \& Judge, P. G. 2000, ApJ, 531,1150

von Uexküll, M., \& Kneer, F. 1995, A\&A, 294, 252

Worden, J., Harvey, J., \& Shine, R. 1999, ApJ, 523, 450 